\title{
Generation of Eupnea and Sighs by a Spatiochemically Organized Inspiratory Network
}

\author{
Araya Ruangkittisakul, ${ }^{1}$ Stephan W. Schwarzacher, ${ }^{2}$ Lucia Secchia, ${ }^{1}$ Yonglie Ma, ${ }^{1}$ Nicoleta Bobocea, ${ }^{1}$ Betty Y. Poon, ${ }^{1}$ \\ Gregory D. Funk, ${ }^{1}$ and Klaus Ballanyi ${ }^{1}$ \\ ${ }^{1}$ Department of Physiology and Perinatal Research Centre, University of Alberta, Edmonton, Alberta, Canada T6G 2S2, and ${ }^{2}$ Department of Anatomy and \\ Neuroanatomy, University of Frankfurt, 60590 Frankfurt/Main, Germany
}

The discovery of the rhythmogenic pre-Bötzinger complex (preBötC) inspiratory network, which remains active in a transverse brainstem slice, greatly increased the understanding of neural respiratory control. However, basic questions remain unanswered such as (1) What are the necessary and sufficient slice boundaries for a functional preBötC? (2) Is the minimal preBötC capable of reconfiguring between inspiratory-related patterns (e.g., fictive eupnea and sighs)? (3) How is preBötC activity affected by surrounding structures? Using newborn rat slices with systematically varied dimensions in physiological $\left[\mathrm{K}^{+}\right](3 \mathrm{mM})$, we found that a $175 \mu \mathrm{m}$ thickness is sufficient for generating inspiratory-related rhythms. In 700- $\mu \mathrm{m}$-thick slices with unilaterally exposed preBötC, a kernel $<100 \mu \mathrm{m}$ thick, centered $0.5 \mathrm{~mm}$ caudal to the facial nucleus, is necessary for rhythm generation. Slices containing this kernel plus caudal structures produced eupneic bursts of regular amplitude, whereas this kernel plus rostral tissue generated sighs, intermingled with eupneic bursts of variable amplitude ("eupnea-sigh pattern"). After spontaneous arrest of rhythm, substance-P or neurokinin-1 (NK1) receptor agonist induced the eupnea-sigh burst pattern in $\geq 250$ - $\mu \mathrm{m}$-thick slices, whereas thyrotropin-releasing hormone or phosphodiesterase- 4 blockers evoked the eupnea burst pattern. Endogenous rhythm was depressed by NK1 receptor antagonism. Multineuronal Ca ${ }^{2+}$ imaging revealed that preBötC neurons reconfigure between eupnea and eupnea-sigh burst patterns. We hypothesize a (gradient-like) spatiochemical organization of regions adjacent to the preBötC, such that a small preBötC inspiratory-related oscillator generates eupnea under the dominant influence of caudal structures or thyrotropin-releasing hormone-like transmitters but eupnea-sigh activity when the influence of rostral structures or substance-P-like transmitters predominates.

Key words: eupnea; histology; brain stem; pre-Bötzinger; respiratory rhythm; $\mathrm{Ca}^{2+}$ imaging

\section{Introduction}

The pre-Bötzinger complex (preBötC) contains neurons that are necessary for generating inspiratory-related rhythm and remain active in a transverse brainstem slice from perinatal rodents (Smith et al., 1991). Since its first description, numerous in vitro and in vivo reports support the hypothesis that the preBötC is a pivotal rhythmogenic inspiratory network (Ramirez et al., 2002; Feldman and Del Negro, 2006). Despite the importance of the preBötC discovery for the understanding of neural respiratory control, fundamental questions remain unanswered. For example, the rostrocaudal boundaries of regions necessary and sufficient for generation of inspiratory-related behaviors in preBötC slices have not been defined. Furthermore, it is proposed that the preBötC can reconfigure between distinct inspiratory-related patterns, including fictive eupnea- or sigh-like activities, in slices

Received April 27, 2007; revised Jan. 14, 2008; accepted Jan. 15, 2008.

This work was supported by the Canadian Institutes of Health Research (CIHR), the Alberta Heritage Foundation for Medical Research (AHFMR), the Canadian Foundation for Innovation, and the Alberta Innovation and Research Investments Program. K.B. and G.D.F. are an AHFMR Scientist and Senior Scholar, respectively. A.R. has been awarded a CIHR studentship (Maternal-Fetal-Newborn training grant).

Correspondence should be addressed to Dr. Klaus Ballanyi at the above address. E-mail: klaus.ballanyi@ualberta.ca.

DOI:10.1523/JNEUROSCI.1926-07.2008

Copyright $\odot 2008$ Society for Neuroscience $\quad 0270-6474 / 08 / 282447-12 \$ 15.00 / 0$
$>500 \mu \mathrm{m}$ thick (Lieske and Ramirez, 2006a,b; Tryba et al., 2006). It is not known whether the preBötC retains its capacity for reconfiguration when isolated in a thinner slice. Alternatively, the reconfiguration may be driven by more rostral or caudal structures. Specifically, the preBötC is neighbored and presumably influenced by other respiratory groups, such as the parafacial respiratory group/retrotrapezoid nucleus complex (Onimaru and Homma, 2003; Mulkey et al., 2004) and nonrespiratory modulatory areas such as the raphe (Richerson, 2004; Feldman and Del Negro, 2006). If the presumptive capability of the preBötC to generate different inspiratory-related behaviors depends on input from rostrocaudally neighboring structures, it is possible that slices lacking these structures cannot produce such activities. Here, we tested this hypothesis and also assessed basic features of inspiratory-related activity such as burst rates and longevity of rhythm in solution of physiological $(3 \mathrm{~mm})\left[\mathrm{K}^{+}\right]$in slices with systematically varied boundaries and thickness using “on-line histology" (Ruangkittisakul et al., 2006).

We found that the minimal preBötC slice thickness that is sufficient for generation of inspiratory rhythm is $175 \mu \mathrm{m}$, whereas a kernel of rhythmogenic tissue $<100 \mu \mathrm{m}$ appears to be necessary for bursting in $700 \mu \mathrm{m}$ slices with the preBötC exposed to one surface. The latter slices generated fictive eupnea bursts with regular amplitude and frequency when the preBötC was isolated with caudal tis- 
sue. Conversely, fictive sighs intermingled with rhythmic activity of variable amplitude, but burst duration typical for eupnea, were generated in $700 \mu \mathrm{m}$ slices containing the preBötC plus rostral tissue. In these preparations, and in 250-500 $\mu \mathrm{m}$ slices with the preBötC in the center, thyrotropin-releasing hormone (TRH) and cAMPdependent phosphodiesterase- 4 blockers induced the eupnea burst pattern after spontaneous arrest of rhythm ("in vitro apnea"), whereas substance-P (SP) and neurokinin-1 (NK1) receptor agonist evoked the eupnea-sigh burst pattern. Multicellular $\mathrm{Ca}^{2+}$ imaging revealed that preBötC neurons active during eupnea are active also during the eupnea-sigh burst pattern. This supports the previous hypothesis that these behaviors derive from preBötC reconfiguration (Lieske et al., 2000).

We hypothesize that a small rhythmogenic preBötC kernel can reconfigure to produce either a eupnea or a eupnea-sigh inspiratory-related burst pattern in physiological $\left[\mathrm{K}^{+}\right]$. The former emerges when the influence of caudal structures or TRHlike transmitters is dominant, whereas the latter emerges when the influence of rostral structures or SP-like transmitters is dominant.

\section{Materials and Methods}

Preparation and solutions. Experiments were performed on brainstem slices from Sprague Dawley (S-D) and Wistar (W) rats between postnatal day $0(\mathrm{P} 0)$ and $\mathrm{P} 4$. All procedures were performed in compliance with the guidelines of the Canadian Council for Animal Care and with the approval of the University of Alberta Health Animal Care and Use Committee for Health Sciences. Rats were anesthetized with isoflurane until the paw withdrawal reflex disappeared. They were then decerebrated, and the neuraxis was isolated at $19-22^{\circ} \mathrm{C}$ in superfusate containing (in mм) $120 \mathrm{NaCl}, 3 \mathrm{KCl}, 1 \mathrm{CaCl}_{2}, 2 \mathrm{MgSO}_{4}, 26 \mathrm{NaHCO}_{3}, 1.25 \mathrm{NaH}_{2} \mathrm{PO}_{4}$, and 20 D-glucose, $\mathrm{pH}$ adjusted to 7.4 by gassing with $95 \% \mathrm{O}_{2}, 5 \% \mathrm{CO}_{2}$. For some experiments, superfusate $\left[\mathrm{K}^{+}\right]$was increased by the addition of $\mathrm{KCl}$. After removal of the cerebellum and transection slightly rostral to the caudal cerebellar artery and just rostral to the $\mathrm{C}_{1}$ spinal segment (Fig. $1 A$ ), the brainstem was glued rostral side down to a metal plate, and preBötC slices with defined boundaries were generated using on-line histology (Ruangkittisakul et al., 2006) (Fig. 1B). Serial sections were made with a Vibratome (VT1000S; Leica, Richmond Hill, Ontario, Canada) in the caudorostral direction starting at the caudal end of the inferior olive. Section thickness was reduced to $100 \mu \mathrm{m}$ when approaching the level for generating a rhythmic slice as evidenced by respiratory marker structures, in particular the subnuclei of the inferior olive. These structures in the prerhythmic slices were compared with a newborn rat brainstem atlas to predetermine, on-line, the rostrocaudal position of the prospective rhythmic slice (Ruangkittisakul et al., 2006). When necessary, the thickness of the last prerhythmic slice was $<100 \mu \mathrm{m}$ to produce a rhythmic slice with a specific boundary. For "off-line" analysis of slice boundaries, prerhythmic and postrhythmic "frame" sections were fixed (see below, Agents) and stained with thionin (Fig. 1B).

Boundaries determined previously via surface values in $500-\mu \mathrm{m}$-thick slices with the preBötC in the middle (m-preBötC[500] slices) were very similar to targeted values, although a 50-100 $\mu \mathrm{m}$ gap between the rhythmic slices and the adjacent frame sections had to be taken into account (Ruangkittisakul et al., 2006). Here, boundaries of $<300-\mu \mathrm{m}$-thin $\mathrm{m}$-preBötC slices according to the determination of surface structures were $25-50 \%$ smaller than tissue thickness. The physical thickness of $200-\mu \mathrm{m}$-thin sectioned agar blocks or living slices cut in superfusate indicated that the error of vibratome cutting was $<8 \%$. Thus, the mean values between slice surface and frame were used instead of the slice surface value alone to identify the boundaries in $<300-\mu \mathrm{m}$-thick slices. In addition to m-preBötC slices of $175-700 \mu \mathrm{m}$ thickness, $700-\mu \mathrm{m}$-thick slices containing either "rostral" brainstem tissue plus the preBötC ( $\mathrm{r}+$ preBötC slices) or "caudal" tissue plus the preBötC $(\mathrm{c}+$ preBötC slices) were used. The complete label for slices included two numbers; the first indicates their thickness, and the second indicates the individual or mean distance from the caudal margin of the facial (VII) motor nucleus

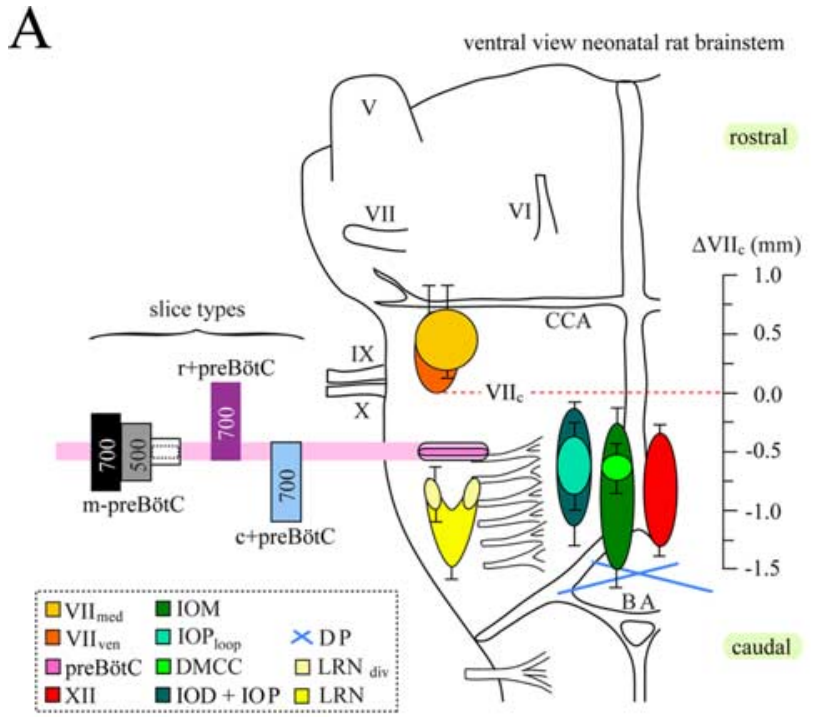

B

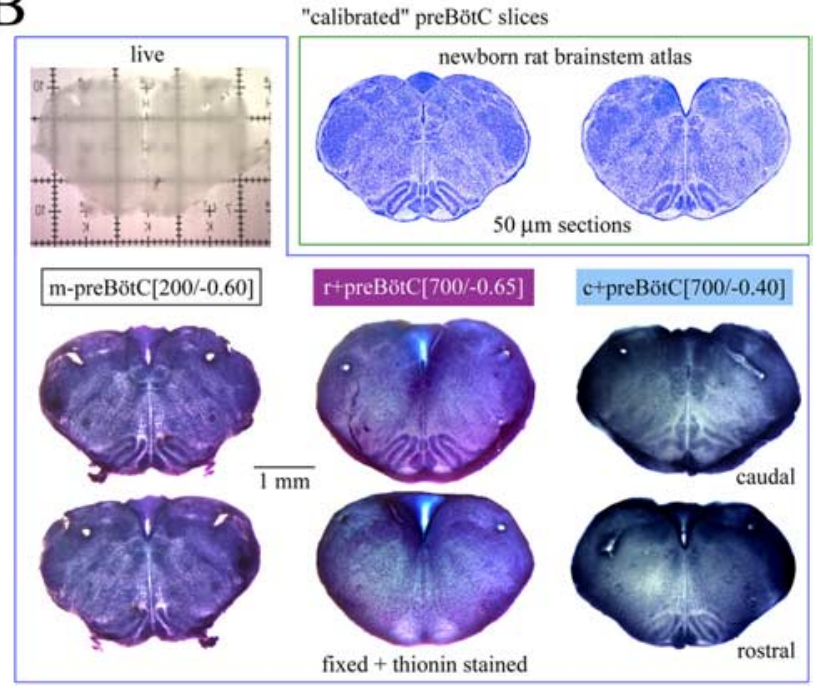

Figure 1. On-line histology for the determination of structure-function relationships in physiological $(3 \mathrm{~mm})\left[\mathrm{K}^{+}\right]$of the isolated inspiratory preBötC neuronal network. $A$, Rostrocaudal extensions in postnatal day P0-P4 S-D or W rats of brainstem marker nuclei for generating rhythmic preBötC slices. The data stem from 15 fixed brainstems plus 11 brainstems sectioned in saline used for studying slice functions. The small SDs indicate a high constancy of these nudlei during early postnatal development. The boundaries of preBötC slices areidentified by the distance (in millimeters, negativesign indicating caudal location) from the caudal end of the facial (VII) motor nucleus (VII) by comparing structures in the pre/postrhythmic slices with those in a newborm rat brainstem atlas (Ruangkittisakul et al., 2006) (see B). DMCC, Dorsomedial cell column of inferior olive; 10 , inferior olive; IOM, medial inferior olive; IOD, dorsal inferior olive; IOP, principal inferior olive; IOP ,oop , lateral loop of IOP; LRN, lateral reticular nucleus; $\mathrm{LRN}_{\text {divy }} \mathrm{LRN}$ divided into medial and lateral subnuclei; $\mathrm{VII}_{\text {med }}$ medial subnucleus of $\mathrm{VI} ;$ $\mathrm{VII}_{\text {ven }}$, ventral subnucleus of VII; $; \mathrm{Xll}$, hypoglossal nucleus; $\mathrm{DP}$, pyramidal decussation; $\mathrm{BA}$, basilar arteny; $\mathrm{CCA}$, caudal cerebellar artery; $\mathrm{V}, \mathrm{VI}, \mathrm{IX}$, and X, trigeminal, abducens, glossopharyngeal, and vagal nerves, respectively. $\boldsymbol{B}$, For the determination of regions that are sufficient or necessary for the generation of inspiratoryrelated rhythm, slices were generated of different thickness with the preBötC in the middle ( $m$-preBötC slices), or containing either caudal or rostral tissue plus the preBötC ( $c+$ preBötC and $r+$ preBötC slices) (valuesin bracketsindicateslicethicknessin micrometers and boundanyin millimeters; seealso leftpanel of A). The calibration barisidentical foralls slicesin $\boldsymbol{B}$. Theleftslicein the top rightboxshowsthe -0.65 section from the atlas corresponding to the caudal surface of the $r+$ preBötC[700/ -0.65$]$ slice. The right slice shows the -0.40 section from the atlas corresponding to the rostral boundary of the $\mathrm{c}+$ preBötC[700/ $-0.40]$ slice.

(VII $)$. A negative sign represents the location caudal to VII ${ }_{c}$ of the caudal slice boundary. However, for $\mathrm{c}+$ preBötC slices, the value reported is the distance from their rostral boundary to VII $_{c}$ (Fig. 1). The final components of the label refer to rat strain and age. Thus, a $500-\mu \mathrm{m}$-thick slice 
from a $\mathrm{P} 3 \mathrm{~W}$ rat with the preBötC in the middle and a caudal surface 0.71 mm caudal to VII $_{c}$ was labeled m-preBötC[500/-0.71]W-P3. Rhythmic slices were immediately fixed caudal side up (except $\mathrm{c}+$ preBötC slices) with insect pins on the silicone layer covering the bottom of the recording chamber (volume, $1.5 \mathrm{ml}$ ). Superfusate was administered at a flow rate of $5 \mathrm{ml} / \mathrm{min}$ via a peristaltic pump (Watson-Marlow Alitea-AB; Sin-Can, Calgary, Alberta, Canada). Superfusate temperature in the recording chamber was $25-27^{\circ} \mathrm{C}$ (TC-324B; Harvard Apparatus, Saint-Laurent, Quebec, Canada).

Agents. Drugs and solutions included the following: TRH (0.5-100 nм; stocks, $1 \mu \mathrm{M}$ and $1 \mathrm{~mm}$ in $\mathrm{H}_{2} \mathrm{O}$ ), GR82334 (2.5 $\mu \mathrm{M}$; stock, $1 \mathrm{~mm}$ in $\mathrm{H}_{2} \mathrm{O}$ ), GR73632 (2.5-10 nM; stock, 1 mm in $\mathrm{H}_{2} \mathrm{O}$ ), SP (25-100 nм; stock, $1 \mathrm{~mm}$ in dimethylsulfoxide), rolipram ( $1 \mu \mathrm{M}$; stock, $1 \mathrm{~mm}$ in dimethylsulfoxide), theophylline (2.5 mM; added to superfusate), fluo-4-AM (0.5 mM; stock, $5 \mathrm{~mm}$ in dimethylsulfoxide with $20 \%$ pluronic acid), agar-agar $(6 \%$ in $\left.\mathrm{H}_{2} \mathrm{O}\right), 4 \%$ paraformaldehyde fixation solution in phosphate buffer (1:2 mixture of $0.1 \mathrm{M} \mathrm{NaH}_{2} \mathrm{PO}_{4}$ plus $0.1 \mathrm{M} \mathrm{Na}_{2} \mathrm{HPO}_{4}$ in $\mathrm{H}_{2} \mathrm{O}$, pH 7.2), and staining solution ( $1 \%$ thionin acetate in $0.1 \mathrm{~m}$ sodium acetate trihydrate plus $0.1 \mathrm{M}$ acetic acid). Agents were obtained from Sigma-Aldrich (Oakville, Ontario, Canada), except salts for the superfusate, sodium acetate trihydrate, and acetic acid (Fisher Scientific, Ottawa, Ontario, Canada); theophylline and glutamate (ICN Biomedicals, Costa Mesa, CA); fluo-4-AM (TEF Labs, Austin, TX); and GR82334 and GR73632 (Tocris Biosciences, Ellisville, $\mathrm{MO}$ ).

Histological procedures. For staining, frame sections were sequentially incubated (after $>15 \mathrm{~min}$ of fixation) in phosphate buffer ( $2 \mathrm{~min}$ ), thionin solution ( $45 \mathrm{~s}$ ), phosphate buffer ( $2 \mathrm{~min}), 50 \%$ ethanol ( $4 \mathrm{~min})$, and finally, phosphate buffer $(2 \mathrm{~min})$. Sections were then transferred on a hanging drop glass slide (Fisher Scientific) to a microscope (Standard 16; magnification, $32 \times$; Zeiss, Jena, Germany) and photographed (PLA642-1.3 Megapixel; PixeLINK, Ottawa, Ontario, Canada). After the experiments, rhythmic slices were fixed overnight and stained using the same procedure, except these thicker slices were immersed in thionin for 90 s. Stained rhythmic slices were photographed (PL-A686-6.6 Megapixel; PixeLINK) in phosphate buffer under a stereo microscope (Zeiss SR15; magnification, 32×) (Fig. $1 B$ ).

Electrophysiological recording. Neuronal population activities were differentially recorded (DAM 50; WPI, Sarasota, FL) via suction electrodes (outer diameter, $80-250 \mu \mathrm{m}$ ) filled with superfusate. Electrodes were positioned in the ventrolateral regions of the slices (supplemental Figs. S1, S2, available at www.jneurosci.org as supplemental material) for (simultaneous) monitoring of bursting within the bilaterally organized ventral respiratory column that includes the preBötC (Alheid et al., 2004). Signals were amplified (10,000 times), bandpass filtered (0.3-3 $\mathrm{kHz}$ ), integrated, and digitally recorded at a sampling rate of $1-4 \mathrm{kHz}$ (Powerlab/8SP; ADInstruments, Colorado Springs, CO).

Multiphoton/confocal $\mathrm{Ca}^{2+}$ imaging. The activity of multiple preBötC neurons was simultaneously assessed with $\mathrm{Ca}^{2+}$ imaging (Ruangkittisakul et al., 2006). The membrane-permeant, $\mathrm{Ca}^{2+}$-sensitive dye fluo4-AM was backfilled into a broken (5-10 $\mu \mathrm{m}$ outer diameter) patch pipette and pressure injected (0.7-1.0 psi, $10 \mathrm{~min})$ into the preBöt $C$ while inspiratory rhythm was monitored electrophysiologically from the contralateral preBötC. Fluorescence signals were assessed with a confocal microscope (Olympus FV300, Fluoview software; Carsen Group, Markham, Ontario, Canada) or a FV300 connected to a Ti:Sa laser (Coherent, Santa Clara, CA) for multiphoton imaging. $\mathrm{Ca}^{2+}$ oscillations, cell bodies, and primary dendrites could be resolved in areas of $200-300 \mu \mathrm{m}$ diameter at tissue depths up to $60 \mu \mathrm{m}$ for confocal and $90 \mu \mathrm{m}$ for multiphoton microscopy. Rhythmic $\mathrm{Ca}^{2+}$ rises were visualized as increases in fluo-4-AM fluorescence intensity in up to 15 cells (typically 3-8 cells) per single $x y$-image plane. Stained regions were monitored using a $2-3 \times$ digital zoom at reduced settings for $y$-axis scanning. Compared with full-frame acquisition $(512 \times 512$ pixels $)$, such "clipped mode" imaging sampled an area of $512 \times 100-220$ pixels and provided scan rates of $1.25-1.43$ scans/s, sufficient to detect $70-100 \%$ of the peak of inspiratory-related $\mathrm{Ca}^{2+}$ rises.

Data analysis. The inspiratory burst rate was averaged every $20 \mathrm{~min}$ over 2 min time windows. The "longevity" of inspiratory rhythms was defined as the time from start of the continuous recording until the time when the period between consecutive bursts exceeded $5 \mathrm{~min}$. The rise time of bursts was defined using ClampFit software (Molecular Devices, Chicago, IL) as the time interval from when the signal increased above a threshold set at $10-100 \%$ of the peak amplitude for that burst. Burst duration, defined as the time period from when the signal exceeded to the point when it fell below the $50 \%$ value of the peak amplitude ("halfwidth"), and rate were analyzed at both 20 and 80 min after the start of recording (see above) by averaging $>10$ consecutive bursts (except for sighs, in which at least three events were averaged). Irregularity scores for burst amplitudes were determined for each cycle using the formula $S_{n}=$ $100 \times \operatorname{ABS}\left(X_{n}-X_{n-1}\right) / X_{n-1}$, where $S_{n}$ is the irregularity score for the $n$th cycle, $\mathrm{ABS}$ is the absolute value, and $X_{n}$ and $X_{n-1}$ are peak amplitude of the $n$th cycle and the previous cycle, respectively. Frequency irregularity scores were assessed by the same formula with $X$ representing the time interval between bursts. For the determination of signal-to-noise ratios, the peak amplitude of an inspiratory burst was normalized to the thickness of the trace of suction electrode recording in the absence of rhythmic bursting. Pharmacologically reactivated rhythms were described by averaging burst rates over a $2 \mathrm{~min}$ time period at steady state (i.e., when drug had evoked a maximal and stable effect). Values are means \pm SEM, except for histology (means $\pm \mathrm{SD}$ ). Significance $\left({ }^{*} p<0.05 ;{ }^{* *} p<0.01\right.$ ) was determined with Student's $t$ tests using SigmaPlot (Systat Software, Point Richmond, CA).

Anatomical preconditions for determination of necessary and sufficient preBötC boundaries. The original report on the discovery of the preBötC estimated that its rostrocaudal extension necessary for the generation of inspiratory rhythm spans $\sim 225 \mu \mathrm{m}$ (Smith et al., 1991). This value was derived based on regions necessary for the generation of inspiratoryrelated nerve bursts that were common to rhythmic newborn rat brainstem-spinal cord preparations serially sectioned in either rostrocaudal or caudorostral directions (Smith et al., 1991). Based on the analysis of 15 fixed brainstems, we have recently produced a brainstem atlas for neonatal P0-P4 S-D and W rats (Ruangkittisakul et al., 2006), thereby covering the age range most commonly used for preBötC slices from this species (Smith et al., 1991; Funk et al., 1993; Rekling and Feldman, 1998). In our previous study, the relative positions of respiratory brainstem marker nuclei were plotted in a sagittal plane in relation to the preBötC, the center of which was hypothesized to be located $\sim 0.5 \mathrm{~mm}$ caudal to $\mathrm{VII}_{c}$. Here, we analyzed an additional 11 unfixed brainstems kept during slicing ( $100 \mu \mathrm{m}$ sections) in the same type of saline used to study preBötC slice functions. The mean values $\pm \mathrm{SD}$ of the extensions of respiratory marker nuclei in these preparations were identical to those of the fixed brainstems showing that fixation artifacts were minimal for the generation of the reference atlas. The mean values \pm SD of the rostrocaudal extensions of marker nuclei in all 26 preparations were reported in relation to $\mathrm{VII}_{\mathrm{c}}$ and projected on the ventral brainstem surface to show the anatomical content and borders, referred to $\mathrm{VII}_{c}$, of the different types of preBötC slices with systematically varied boundaries and/or thicknesses (Fig. 1A). Specifically, these were 175 - to $700-\mu \mathrm{m}$-thick m-preBötC, $\mathrm{r}+$ preBötC[700], and c+preBötC[700] slices. These slices were generated with on-line histology, and their boundaries were determined after fixation and thionin staining after the experiments (Fig. $1 B$ ).

Criteria for identification and classification of inspiratory-related bursting. In the present study, we determined the rostrocaudal preBötC boundaries that are necessary and sufficient for the generation of inspiratory-related behaviors in $3 \mathrm{mM} \mathrm{K}^{+}$solution in brainstem slices with systematically varied boundaries and/or thickness (Fig. 1). We also tested the hypothesis that the capability of the preBötC to generate distinct inspiratory-related behaviors is differentially influenced by input from rostrocaudally neighboring structures. Before this, the baseline inspiratory burst behavior (e.g., duration, rise time) of "reference" slices with an undisturbed preBötC was established to facilitate comparison of burst parameters with those generated by the novel slices with exposed (and reduced) preBötC. These 500- and 700- $\mu$ m-thick m-preBötC slices generated three types of inspiratory activities in oxygenated $3 \mathrm{~mm} \mathrm{~K}$ solution [(1) "eupnea (bursts)," (2) "sigh (bursts)," and (3) "biphasic bursts"], as reported for newborn mouse or rat slices (Lieske et al., 2000; Ruangkittisakul et al., 2006) or newborn rat brainstem-spinal cords (Shvarev et al., 2003). The burst patterns comprised either a uniform eupnea 
burst pattern or a pattern in which eupneic bursts were intermingled with either sighs ("eupnea-sigh burst pattern") or biphasic bursts ("eupneabiphasic burst pattern").

Properties that discriminate these different burst patterns are described in the supplemental material (available at www.jneurosci.org). In brief, a burst was counted as a sigh compared with eupneic bursts in the same slice if it had (1) a greater amplitude, (2) a longer duration, (3) a longer rise time, and (4) postsigh inhibition (of burst rate and/or amplitude). Conversely, a burst was counted as a biphasic burst if, compared with eupneic bursts in the same slice, it had (1) a longer duration and (2) a secondary peak that was similar in amplitude to the first peak.

\section{Results}

The rostrocaudal preBötC boundaries that are sufficient and necessary for the generation of inspiratory-related behaviors in $3 \mathrm{~mm}$ $\mathrm{K}^{+}$were determined in preBötC slices by systematically varying their boundaries and/or thickness (Fig. 1). This approach included the analysis of whether distinct inspiratory-related burst patterns including fictive eupnea and sighs depend on slice dimensions. In addition, multiphoton/confocal $\mathrm{Ca}^{2+}$ imaging was used to test the previous hypothesis that the preBötC reconfigures between distinct inspiratory-related burst patterns.

\section{Slice thickness sufficient for inspiratory-related rhythm in 3 $\mathrm{mM} \mathrm{K}^{+}$}

First, we tested whether $250 \mu \mathrm{m}$ or thinner m-preBötC slices generate inspiratory rhythm in $3 \mathrm{~mm} \mathrm{~K}^{+}$. In eight $\mathrm{m}$-preBötC[250/-0.65] slices, rhythmic bursts had maximal amplitudes in the typical ventrolateral respiratory surface region (supplemental Fig. S2, available at www.jneurosci.org as supplemental material), and rhythm lasted $99 \pm 14 \mathrm{~min}$ (Fig. $2 A)$. In contrast, rhythmic bursting was seen neither in this "hot spot" nor surrounding regions in six other $250 \mu \mathrm{m}$ slices with borders deviating $\geq 100 \mu \mathrm{m}$ from the mean margin of the rhythmic slices (Fig. 2A). Similar bursting was seen for $103 \pm 13 \mathrm{~min}$ exclusively in the same respiratory region of six m-preBötC[200/ -0.63 ] slices (Fig. $2 A$ and supplemental Fig. S2, available at www. jneurosci.org as supplemental material). Also, two $\mathrm{m}$-preBötC[175] slices showed rhythmic bursting in the respiratory hot spot, in contrast to lack of rhythmic bursting in this or surrounding regions in five other $175 \mu \mathrm{m}$ slices with similar boundaries (Fig. 2A). The most typical bursts in the $\mathrm{m}$-preBötC[250] slices had a rather uniform amplitude with a good signal-to-noise ratio $(4.2 \pm 0.5 ; n=4)$ (Fig. $2 B-D$ and supplemental Fig. S4, available at www.jneurosci.org as supplemental material). In $>50 \%$ of these slices, the maximal amplitude and rate of such bursts developed over a time period of $\sim 20$ min after the start of recording. At this time, the rate of these bursts was $4.3 \pm 0.7$ bursts $/ \mathrm{min}(n=4)$ and remained constant for the next hour $(2.7 \pm 0.8$ bursts $/ \mathrm{min}$ at $80 \mathrm{~min}$ ) (Fig. $2 \mathrm{~B}$ and supplemental Fig. S5, available at www.jneurosci.org as supplemental material). In six of the eight m-preBötC[250] slices, 2-15 bursts with a smaller and variable amplitude occurred in the interval between the robust bursts at a rate of $8.0 \pm 2.2$ bursts/ $\min (n=4)$ at $20 \mathrm{~min}$ of recording (Fig. $2 C, D)$. Compared with the large-amplitude bursts, the bursts with variable amplitude were significantly smaller at $20 \mathrm{~min}$ (supplemental Fig. S5, available at www.jneurosci.org as supplemental material). At $80 \mathrm{~min}$, a quantitative comparison of both burst types was not possible because of a progressive attenuation of the smaller bursts that started after $>40 \mathrm{~min}$ and was followed by their disappearance (Fig. 2C). The latter features resembled the eupnea-sigh burst pattern in a subgroup of 500- or 600- $\mu \mathrm{m}$-thick m-preBötC slices (supplemental Figs. S3, S4, available at www.jneurosci.org as sup- plemental material). The large bursts in the m-preBötC[250] slices had a shape similar to sighs in thicker m-preBöt slices and also a significantly longer duration and rise time than the smaller bursts (supplemental Figs. S4, S5, available at www.jneurosci.org as supplemental material). Thus, we propose that this burst pattern is a eupnea-sigh pattern. Also, the burst pattern in four of the six rhythmic m-preBötC[200] slices was characterized by sighlike bursts with a signal-to-noise ratio of $2.7 \pm 0.4(n=4)$ that were intermingled with 2-15 eupneic bursts of significantly smaller amplitude and duration (Fig. $2 E$ and supplemental Fig. $S 4$, available at www.jneurosci.org as supplemental material). As in the $250-\mu \mathrm{m}$-thin slices, it took up to $20 \mathrm{~min}$ until the amplitude and rate of the sighs were maximal, whereas the eupneic bursts started to decrease progressively in amplitude after time periods $>40 \mathrm{~min}$. The rate of eupneic bursts at $20 \mathrm{~min}$ was $7.4 \pm$ 2.6 bursts/min $(n=4)$ compared with a sigh rate of $3.6 \pm 0.8$ bursts/min $(n=4)$ that did not change in the next hour $(5.6 \pm 2.0$ bursts/min) (supplemental Fig. S5, available at www. jneurosci.org as supplemental material). The other two $\mathrm{m}$-preBötC[200] slices and two of the eight m-preBötC[250] slices showed rhythmic activity consisting primarily of sighs (Fig. $2 B, E)$. In all eight $\mathrm{m}$-preBötC[250] and $50 \%$ of m-preBötC[200] slices, all sighs and most eupneic bursts were bilaterally synchronous, at least at 20-50 min (Fig. 2B-E). Asynchronous bilateral activity was seen in the remaining m-preBötC[200] slices.

After onset of in vitro apnea, $6 \mathrm{mM} \mathrm{K}^{+}$restored in all $\mathrm{m}$-preBötC[250] slices bilaterally synchronous bursting resembling the original pattern (Fig. $2 B, C$ ). In three of these slices, the addition to $3 \mathrm{~mm} \mathrm{~K}{ }^{+}$solution of the clinical respiratory stimulant theophylline $(2.5 \mathrm{~mm})$ (Bhatia, 2000) resulted in a eupnea burst pattern. Application of TRH (1-100 nM) and rolipram (1 $\mu \mathrm{M})$ in $3 \mathrm{mM} \mathrm{K}^{+}$during in vitro apnea also evoked a eupnea burst pattern, whereas SP (25-100 nM) or the NK1 receptor agonist GR73632 (2.5-10 nM) elicited bursting with a eupnea-sigh pattern similar to m-preBötC[500] slices (Figs. $2 B-D, 3$ ). Also, in all $\mathrm{m}$-preBötC[200] slices, $6 \mathrm{~mm} \mathrm{~K}^{+}$restored bilaterally synchronous rhythm, the burst amplitude and rate of which were further enhanced and transformed into a eupnea pattern by $2.5 \mathrm{~mm}$ theophylline (Fig. 2E). TRH, rolipram, or SP was not tested, because the capability of $200 \mu \mathrm{m}$ slices to generate rhythm appeared to deteriorate after $>3 \mathrm{~h}$ (i.e., after the test for longevity, $6 \mathrm{~mm} \mathrm{~K}^{+}$, and theophylline). Burst parameters were not quantified in the two active $m$-preBötC[175] slices because the signal-to-noise ratio was too low $(<1.5)$ and bursting was quite irregular in $3 \mathrm{~mm}$ $\mathrm{K}^{+}$. These slices showed rhythmic activity for 33 and $38 \mathrm{~min}$ with a maximal rate of 4.0 and 2.8 bursts/min, respectively. One slice showed bilaterally nonsynchronous bursts, whereas the other generated only unilateral activity. During in vitro apnea, $6 \mathrm{~mm}$ $\mathrm{K}^{+}$(plus $2.5 \mathrm{~mm}$ theophylline) increased the signal-to-noise ratio to $>2$ and elicited synchronous bursts in these slices.

These results showed that slices as thin as $175 \mu \mathrm{m}$ can generate inspiratory-related rhythm in $3 \mathrm{mM} \mathrm{K}^{+}$. The area between $\sim 0.42$ and $0.59 \mathrm{~mm}$ caudal to $\mathrm{VII}_{\mathrm{c}}$, contained in these slices, is also common to rhythmic 200-250 $\mu \mathrm{m}$ slices (Fig. $2 A$ ). The typical burst pattern in most 200- to 250 - $\mu \mathrm{m}$-thin slices resembles the eupnea-sigh pattern in some 500 - or 600 - $\mu \mathrm{m}$-thick m-preBötC slices.

\section{Necessary preBötC boundaries in $700 \mu \mathrm{m}$ slices with exposed preBötC}

Next, inspiratory-related bursting in $3 \mathrm{mM} \mathrm{K}^{+}$was assessed in $700-\mu \mathrm{m}$-thick slices in which the preBötC was exposed, more or less, at either their rostral or caudal surface. Ten $c+$ preBötC[700/ 


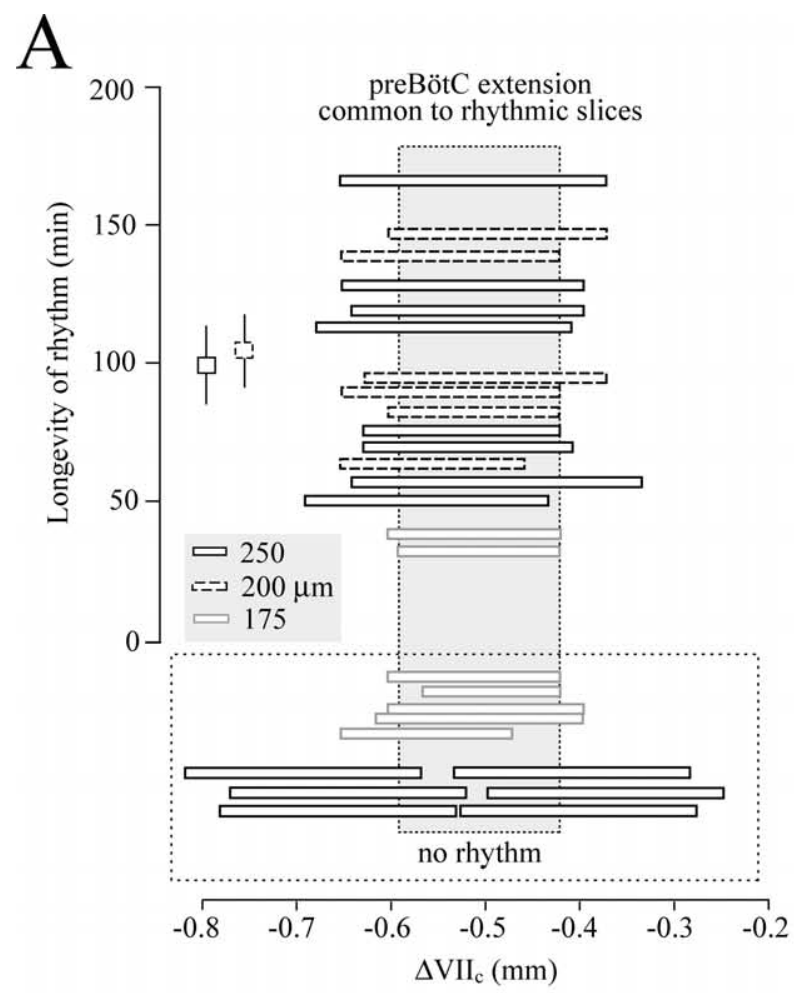

\section{$3 \mathrm{~K}^{+}(60 \mathrm{~min})$

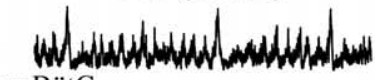 preBötC left

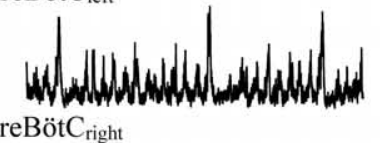 \\ preBötC $\mathrm{r}_{\text {right }}$}

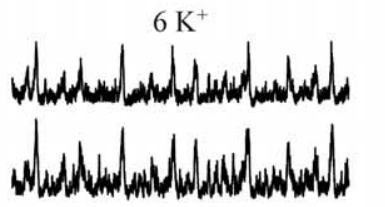

D
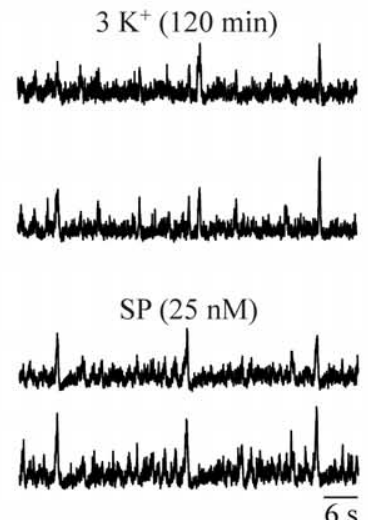

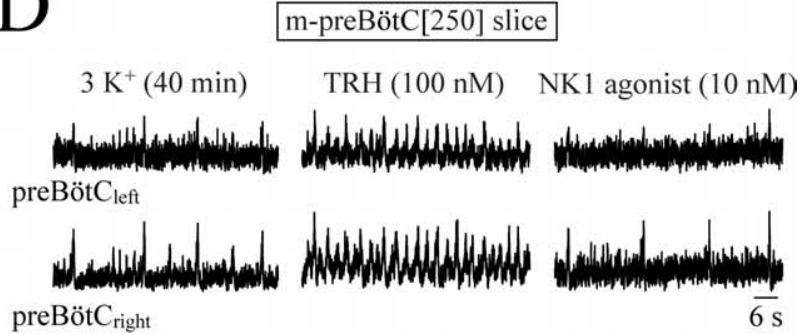

B

m-preBötC[250] slice

E m-preBötC[200] slice
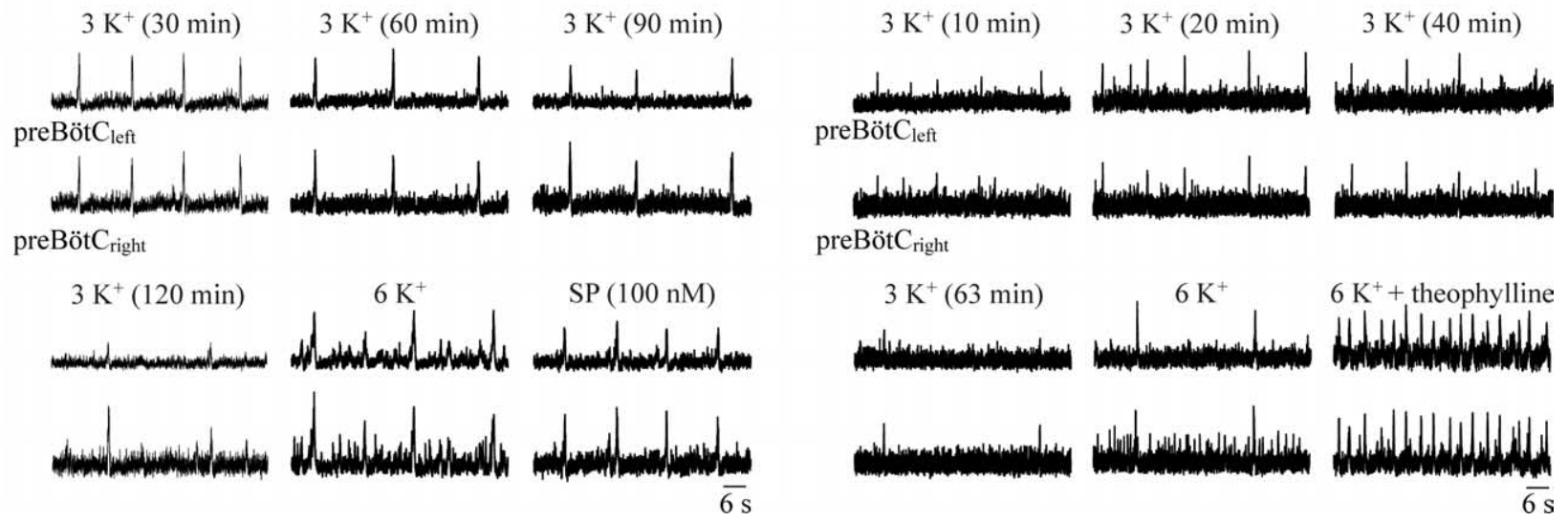

Figure 2. Boundaries and burst properties in $3 \mathrm{~mm} \mathrm{~K}{ }^{+}$of thin m-preBötC slices. $A$, Plot of the rostrocaudal boundaries of eight m-preBötC[250/-0.65]W-P0/1 slices, six m-preBötC[200/ $-0.63] \mathrm{W}-\mathrm{P0}$ slices, and seven m-preBötC[175/-0.63]W-P0 slices versus the longevity of inspiratory-related bursting. The mean ( \pm SEM) of the longevities of rhythms in the 250 - and 200 - $\mu \mathrm{m}$ thin slices are indicated by the squares. The gray box represents the region common to the rhythmic slices. Note that five of seven $\mathrm{m}$-preBötC[175] slices with boundaries similar to those of the two rhythmic slices did not show rhythm. In contrast, the boundaries of six nonrhythmic m-preBötC[250] slices deviated notably from those of the rhythmic slices of this type. $\boldsymbol{B}, A$ m-preBötC[250/ -0.65]W-P1 slice showed bilaterally synchronous robust bursts with a pattern resembling the eupnea-sigh pattern (although with very-small-amplitude eupneic bursts) in thicker slices (compare supplemental Fig. S1, available at www.jneurosci.org as supplemental material). This rhythm was stable for $>90 \mathrm{~min}$, before it stopped spontaneously (in vitro apnea) shortly after $120 \mathrm{~min}$. Sighs and smaller-amplitude eupneic bursts were activated by $6 \mathrm{~mm} \mathrm{~K}^{+}$, whereas SP, after return to $3 \mathrm{~mm} \mathrm{~K}^{+}$solution, evoked primarily sighs. C, A m-preBötC[250/-0.65]W-P1 slice generated bilaterally synchronous bursts resembling the eupnea-sigh pattern in thicker slices (supplemental Figs. S1,S3, available at www.jneurosci.org as supplemental material). The burst pattern became more irregular after $>60 \mathrm{~min}$, partly because of disappearance of the eupneic bursts, but rhythm persisted for an additional $106 \mathrm{~min}$, before rhythm stopped. A $6 \mathrm{~mm} \mathrm{~K}^{+}$solution reactivated both sighs and eupneic bursts, whereas SP elicited sighs and intermittent very-small-amplitude eupneic bursts. D, After in vitro apnea after $113 \mathrm{~min}$ in a m-preBötC[250/-0.68]W-P1 slice, TRH evoked a regular pattern of eupneic bursts, whereas subsequent application after washout of TRH of the NK1 receptor agonist GR73632 evoked sighs at rather low-rate and intermittent very-smallamplitude eupneic bursts. $E$, Twenty minutes after the start of the recording, bilaterally synchronous bursting in the m-preBötC[200/-0.60]W-P0 slice of Figure $1 B$ reached a maximal amplitude. Burst amplitude decreased, and rate slowed after $63 \mathrm{~min}$ to 2 bursts/min and remained at that level for an additional $80 \mathrm{~min}$ before rhythm stopped. Synchronous bursting of sighs and unilateral eupneic bursts were reactivated by $6 \mathrm{~mm} \mathrm{~K}^{+}$, whereas the addition of $2.5 \mathrm{~mm}$ theophylline to $6 \mathrm{~mm} \mathrm{~K}^{+}$solution resulted in a faster rhythm with a eupnea pattern.

-0.42] slices generated rhythmic bursts for $121 \pm 22 \mathrm{~min}$, whereas rhythm lasted $137 \pm 14 \mathrm{~min}$ in $12 \mathrm{r}+$ preBötC[700/ -0.58] slices (Fig. 4A). In both types of slices, burst amplitudes were maximal, with a good signal-to-noise ratio $(>4)$, in the typical respiratory hot spot, even when slice boundaries were $<100 \mu \mathrm{m}$ distant to the proposed location of the preBötC center

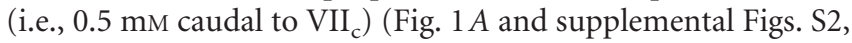
$\mathrm{S} 4$, available at www.jneurosci.org as supplemental material). In 
contrast, nonrhythmic slices did not show any type of phasic bursting in the ventrolateral hot spot or surrounding regions. The rostrocaudal brainstem region common to rhythmic slices of both types was $<100 \mu \mathrm{m}$, centered at $\sim 0.5 \mathrm{~mm}$ caudal to $\mathrm{VII}_{\mathrm{c}}$, and corresponds to the preBötC extent necessary for generation of inspiratory-related rhythm in $700-\mu \mathrm{m}$ thick slices (Fig. 4A).

\section{Distinct inspiratory patterns in $700 \mu \mathrm{m}$} slices with exposed preBötC

Despite similar longevities of rhythms in both types of $700 \mu \mathrm{m}$ slices with the preBötC at one surface, their inspiratory patterns differed notably. These distinct burst patterns were analyzed in 33 $\mathrm{r}+$ preBötC[700] and $20 \mathrm{c}+$ preBötC[700] slices with boundaries $<150 \mu \mathrm{m}$ distant to the center of the preBötC. Fifteen of the $c+$ preBötC[700] slices showed a eupnea burst pattern, whereas a eupnea-biphasic burst pattern was seen in the other five slices (Figs. $4 A, B, 5 A$ ). In contrast, only 4 of the $33 \mathrm{r}+$ preBötC[700] slices (12\%) generated rhythm with either a eupnea or eupnea-biphasic burst pattern (two cases each), whereas the burst pattern included sighs in the other 29 slices ( $88 \%$ ) (Fig. 5A). In the latter group of slices, a stable eupnea-sigh burst pattern was seen in 21 cases $(72 \%)$, whereas the rhythm consisted in the other 8 cases (28\%) of a eupnea-sigh pattern with eupneic bursts of very small amplitude that disappeared in 3 cases after $30-45 \mathrm{~min}$ of recording (Figs. $4 A, C, 5 A$ ).

In six c+ preBötC[700] slices with a eupnea burst pattern, the burst rate fell significantly between 20 and $80 \mathrm{~min}$ of recording, from 9.5 to 6.0 bursts $/ \mathrm{min}$, whereas amplitude increased during that time but duration and rise time did not change (supplemental Figs. S4, S5, available at www.jneurosci.org as supplemental material). In six $\mathrm{r}+$ preBötC[700] slices with a eupnea-sigh burst pattern, the amplitude of eupneic bursts increased significantly between 20 and $80 \mathrm{~min}$, whereas sigh amplitude remained stable (supplemental Fig. S4, available at www.jneurosci.org as supplemental material). Sigh rate decreased significantly from 1.9 to 0.7 bursts/min during that time period, whereas the eupneic burst rate ( 5.1 bursts/min at $20 \mathrm{~min}$ ) did not change (supplemental Fig. S5, available at www.jneurosci.org as supplemental material). Both, the duration and rise time of eupneic bursts ( 0.36 and $0.62 \mathrm{~s}$ at $20 \mathrm{~min}$ ) decreased significantly ( 0.18 and $0.44 \mathrm{~s}$ ) between 20 and $80 \mathrm{~min}$ (supplemental Fig. S5, available at www.jneurosci.org as supplemental material). Neither the duration nor rise time of sighs changed during that time period ( 0.56 and $0.96 \mathrm{~s}$ at $20 \mathrm{~min}$, respectively) (supplemental Fig. S5, available at www.jneurosci.org as supplemental material). Further analysis of the dependence of burst patterns on the location of the preBötC in $700-\mu \mathrm{m}$-thick slices revealed that $\mathrm{r}+$ preBötC[700] slices typically showed a major difference between the amplitudes of sighs and eupneic bursts and among
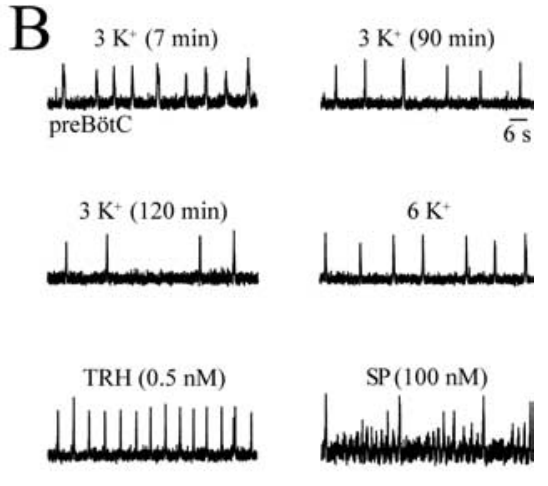
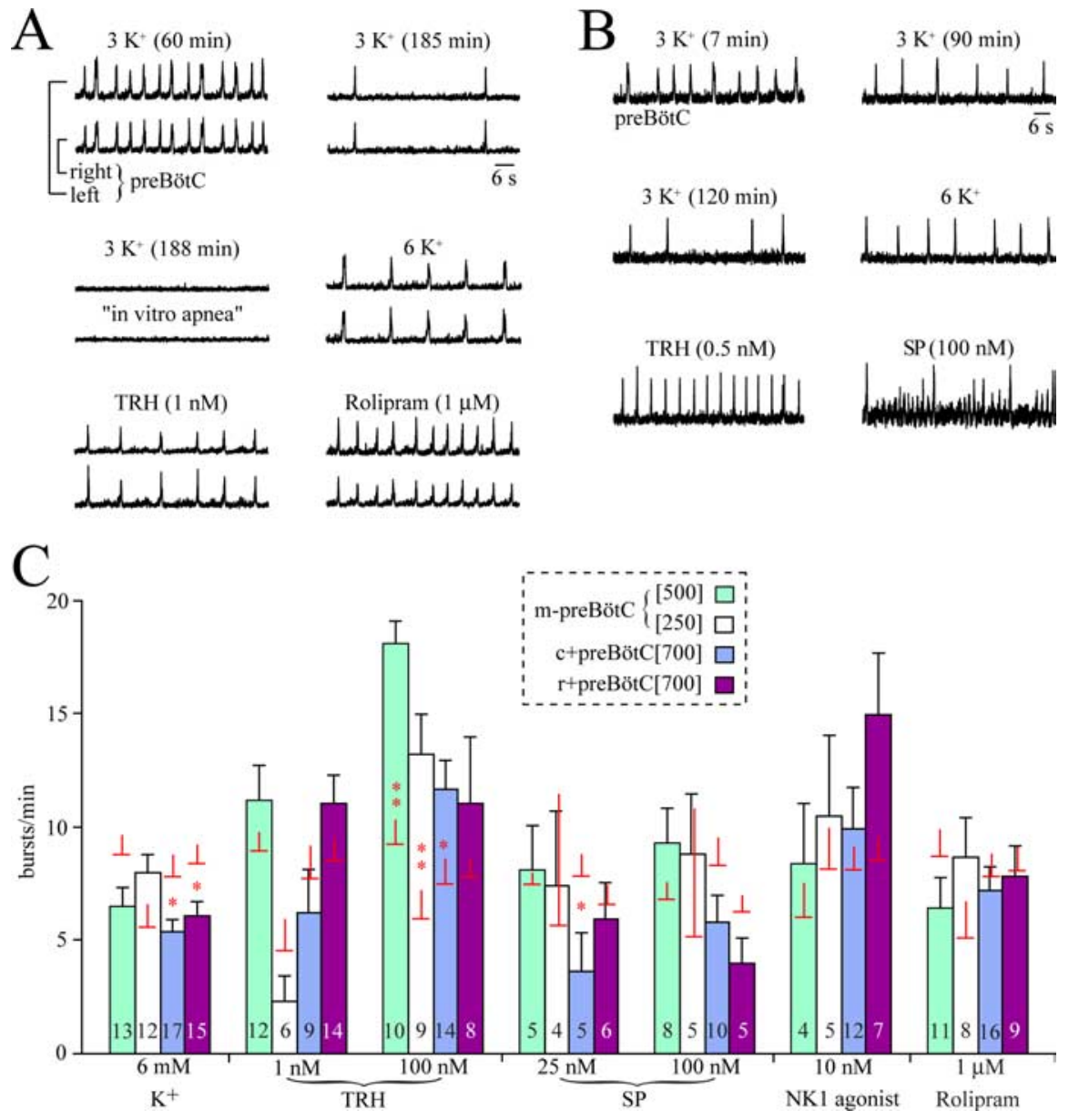

Figure 3. Pharmacological reactivation of $3 \mathrm{~mm} \mathrm{~K}^{+}$rhythms in preBötC slices after onset of in vitro apnea. $\boldsymbol{A}$, In a m-preBötC[500/-0.70]W-P3 slice, the inspiratory rate slowed to 2 bursts/min after $185 \mathrm{~min}$ of recording, and rhythm stopped apnea, bursting was reactivated by $6 \mathrm{~mm} \mathrm{~K}^{+}$, TRH, SP, the NK1 receptor agonist GR73632, and rolipram in the slice types shown dashed box. Bars show means \pm SEM; digits in bars indicate the number of slices. Additional horizontal plus vertical lines indicate the means \pm SEM of the control burst rate for those slices tested for a specific agent. Note that the values correspond to the combined average burst rate of slices that showed a dual burst patterns (i.e., a eupnea- biphasic or a eupnea-sigh pattern).

eupneic bursts (Figs. 4C, 5A). This difference was reflected by an irregularity score of sigh burst amplitudes that was significantly ( $~ 2$ times) higher between 0 and $80 \min (n=8)$ than for eupneic bursts of uniform amplitude in the $\mathrm{c}+$ preBötC[700] slices $(n=$ 8) (Fig. 5B). Similarly, burst rate was significantly more irregular in the $\mathrm{r}+$ preBötC[700] slices than in the $\mathrm{c}+$ preBötC[700] slices (Fig. 5C). Also the rise time of sighs was significantly (approximately two times) longer than that of the eupneic bursts in the c+ preBötC[700] slices (Fig. 5D). After onset of in vitro apnea, TRH $(0.5-100 \mathrm{nM})$ or rolipram $(1 \mu \mathrm{M})$ evoked the eupnea burst pattern, whereas SP $(25-100 \mathrm{nM})$ or NK1 receptor agonist (2.5-10 nM) elicited rhythm with the eupnea-sigh burst pattern in both slice types (Figs. 3, 4B, C).

\section{Effects of NK1 receptor antagonism on preBötC activity}

We found that NK1 receptor activation in vitro induces the eupnea-sigh burst pattern in preBötC-containing medullary slices after onset of in vitro apnea. We thus tested whether NK1 receptors contribute to the generation of endogenous rhythm in $3 \mathrm{mM}$ $\mathrm{K}^{+}$. Bath application of the NK1 receptor antagonist GR82334 


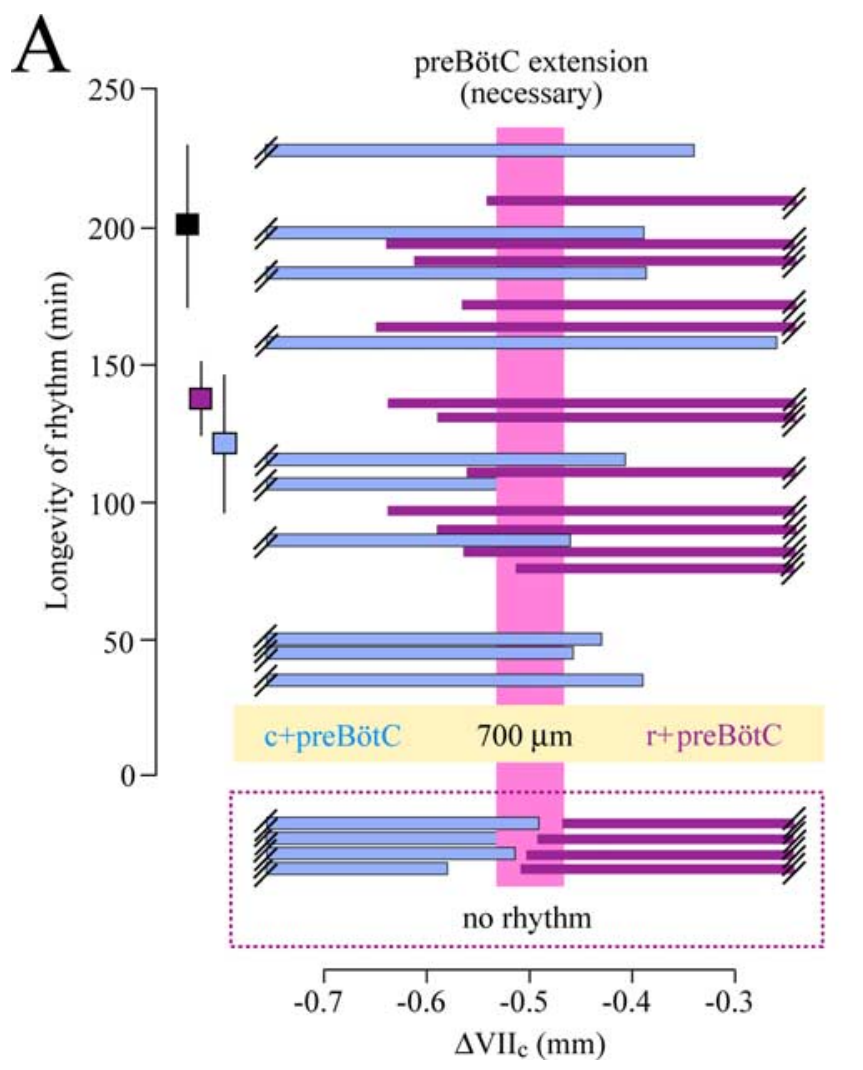

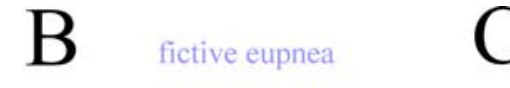
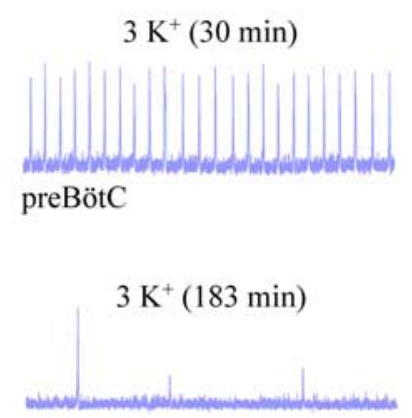

TRH $(100 \mathrm{nM})$
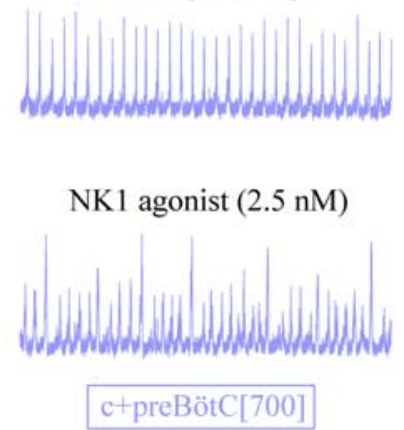
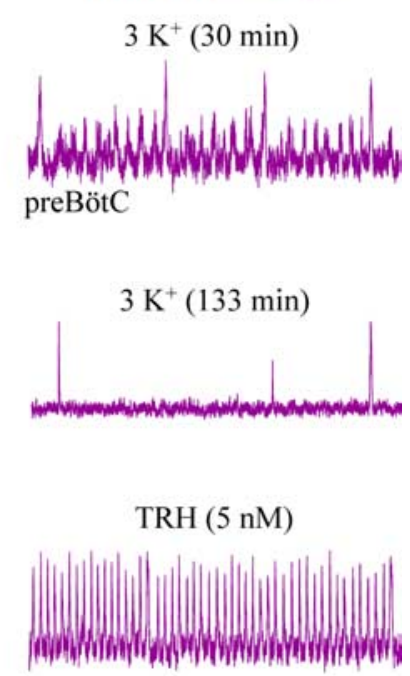

$3 \mathrm{~K}^{+}(133 \mathrm{~min})$

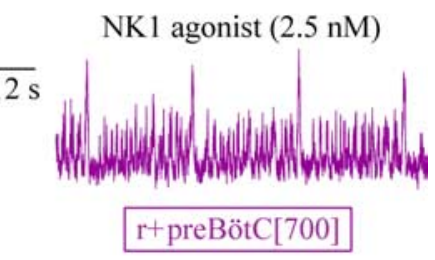

Figure 4. Boundaries necessary for rhythm and activity patterns in $3 \mathrm{~mm}\left[\mathrm{~K}^{+}\right]$of $700-\mu \mathrm{m}$-thick slices with unilaterally exposed preBötC. $A$, Plot of the longevity of rhythms versus boundaries of $10 \mathrm{c}+$ preBötC[700/-0.42]W-P1/4 slices and $12 \mathrm{r}+$ preBötC[700/-0.58]S-D/W-P0/4 slices. The preBötC extension necessary for rhythm is possibly $<100 \mu \mathrm{m}$ as indicated by the pink box framing brainstem tissue common to the rhythmic slices of both types. The dashed box underneath $\boldsymbol{A}$ indicates the boundaries of nonrhythmic slices. The squares next to the longevity scale bar show the mean longevity ( \pm SEM) of rhythms in the $10 \mathrm{c}+$ preBötC[700] (blue square) and the $12 \mathrm{r}+$ preBötC[700] (purple square) slices, whereas the black square shows the longevity of rhythm in 7 m-preBötC[700/-0.89]W-P1/3 slices (see supplemental material, available at www.jneurosci.org as supplemental material). $\boldsymbol{B}$, The top panel shows eupneic bursts of regular rate and amplitude in the $c+$ preBötC[700/-0.40]W-P2 slice of Figure $1 B$. After the occurrence of in vitro apnea $\sim 2$ min after the recording in the second panel from the top, consecutive bath application of TRH (third panel) and (after washout of TRH) NK1 receptor agonist GR73632 (bottom panel) reactivated a eupnea and a eupnea-sigh burst pattern, respectively. C, The top panel shows large sigh bursts and eupneic bursts of smaller and variable amplitude in the $\mathrm{r}+\mathrm{preBöt}[[700 /-0.65] \mathrm{W}-\mathrm{P} 1$ slice of Figure $1 \mathrm{~B}$. After in vitro apnea, occurring $\sim 3$ min after the recording in second panel from the top, TRH induced a eupnea burst pattern (third panel), whereas GR73632 initiated a eupnea-sigh pattern very similar to that in control (bottom panel).

$(2.5 \mu \mathrm{M})$ for 5-15 min abolished the endogenous rhythm within 3-12 min in three of four m-preBötC[350] slices (Fig. 6A,B) and reduced the burst rate to $21 \%$ of control in the fourth case. Within 15-25 min after starting the washout of the antagonist, the mean burst rate recovered to $37 \%$ of control (Fig. $6 A, B$ ). Because recording periods for this protocol lasted $>1 \mathrm{~h}$, the incomplete reversibility may reflect, at least in part, a timedependent reduction in endogenous burst rate.

Also, in five of nine m-preBötC[500] slices, GR82334 blocked the $3 \mathrm{mM} \mathrm{K}^{+}$rhythm within 5-25 min after the start of the bath application. In two of these nine slices, the NK1 receptor antagonist decreased burst rates to 38 and $31 \%$ of control, whereas it had no effect in the remaining two cases. Recovery was maximal within 12-40 min after the start of washout of GR82334 but reached only $35 \%$ of control, similar to the incomplete recovery in $350 \mu \mathrm{m}$ slices (Fig. 6B). We thus tested whether this reflects an incomplete reversibility of GR82334. For this purpose, rhythm with a eupnea-sigh burst pattern was induced by bath application of GR73632 (5 nM) after onset of in vitro apnea in two $\mathrm{m}$-preBötC[350] and two m-preBötC[500] slices (Fig. 6C,D). In all these slices, GR82334 (2.5 $\mu \mathrm{M})$ abolished bursting within 3-14 min, but burst rate recovered very close to its initial rate of $14 \pm$ 1.2 bursts/min within 6-22 min after the washout of GR82334 in the presence of the NK1 receptor agonist (Fig. $6 D, E$ ).

\section{$\mathrm{Ca}^{2+}$ imaging of reconfiguring preBötC neurons}

Finally, we assessed with multiphoton/confocal $\mathrm{Ca}^{2+}$ imaging $(\mathrm{Ru}-$ angkittisakul et al., 2006) whether both eupneic and eupnea-sigh burst patterns derive from separate or rather one reconfiguring preBötC network. In $16 \mathrm{r}+$ preBötC[700] slices with the mean border $110 \mu \mathrm{m}$ caudal to the preBötC center, multineuronal activity was monitored during transitions from the eupnea-sigh burst pattern in $3 \mathrm{mM} \mathrm{K}^{+}$to a eupnea pattern induced during in vitro apnea by TRH (1-100 nM), rolipram ( $1 \mu \mathrm{M})$, or theophylline $(2.5 \mathrm{mM})$. Transitions in burst amplitude and rate, recorded electrophysiologically in one preBötC area, were correlated with $\mathrm{Ca}^{2+}$ transients in all inspiratory preBötC neurons imaged in the contralateral area $40-70 \mu \mathrm{m}$ below the caudal slice surface. Specifically, $88 \%$ (TRH, $n=68$ ), $86 \%$ (rolipram, $n=36$ ), and $86 \%$ (theophylline, $n=32$ ) of preBötC neurons active during the endogenous eupnea-sigh burst pattern reconfigured to a eupnea pattern in response to the three agents (Fig. 7A). The other cells did not show rhythmic $\mathrm{Ca}^{2+}$ rises in response to the drugs, or to subsequent application of 6 or $9 \mathrm{mM} \mathrm{K}^{+}$, indicating phototoxic cell damage (Yuste et al., 2006).

In four different $\mathrm{r}+$ preBötC[700] slices with their surface $\sim 50 \mu \mathrm{m}$ caudal to the preBötC center, GR73632 was used to reactivate a eupnea-sigh burst pattern of preBötC activity after onset of in vitro apnea. The NK1 receptor agonist induced inspiratory-related $\mathrm{Ca}^{2+}$ oscillations corresponding to a eupnea- 
A

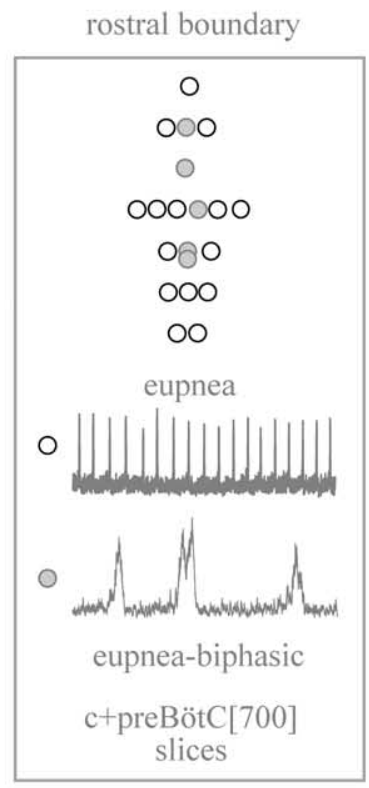

$\mathrm{B}$
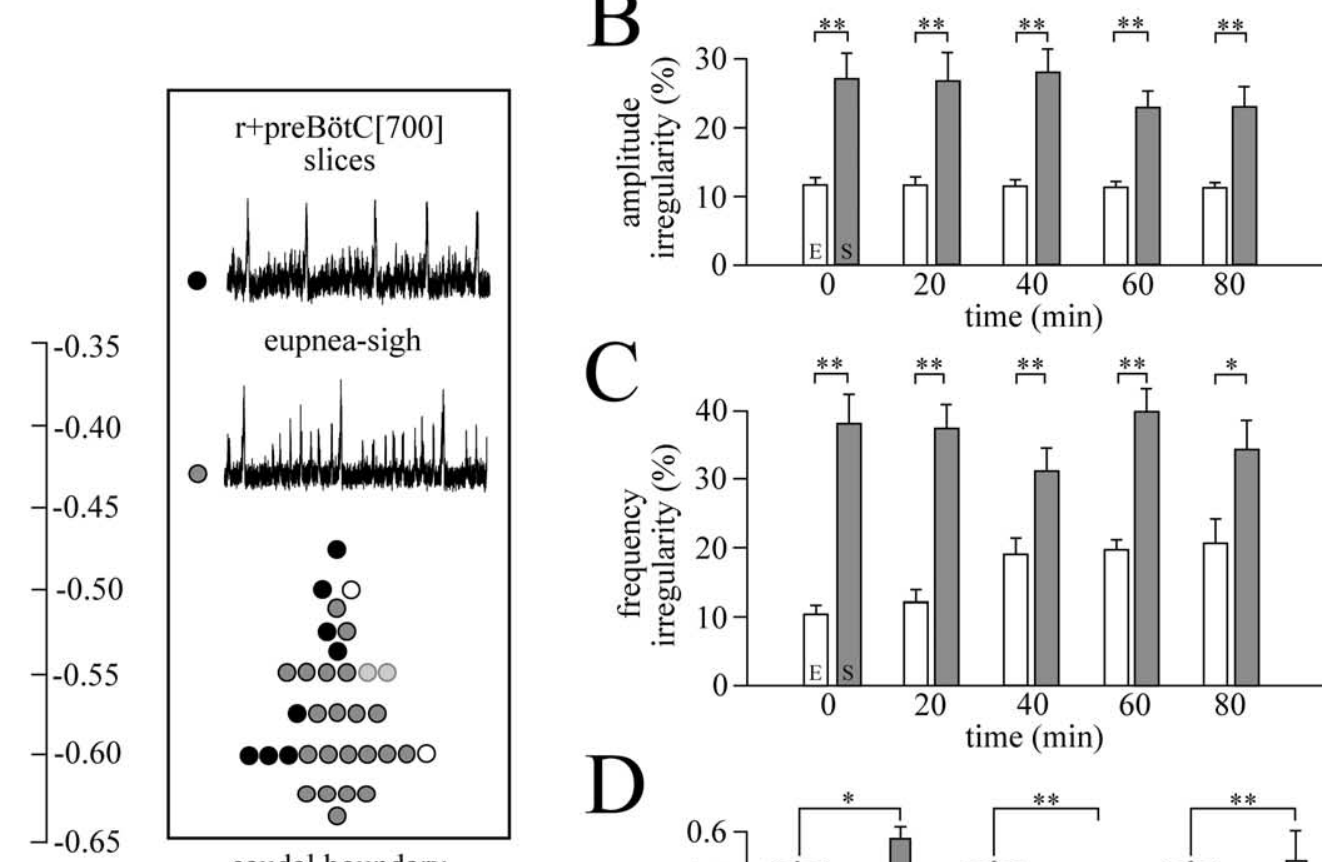

$\Delta \mathrm{VII}_{\mathrm{c}}(\mathrm{mm})$

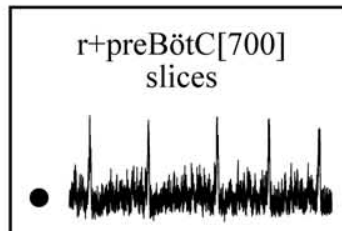

eupnea-sigh

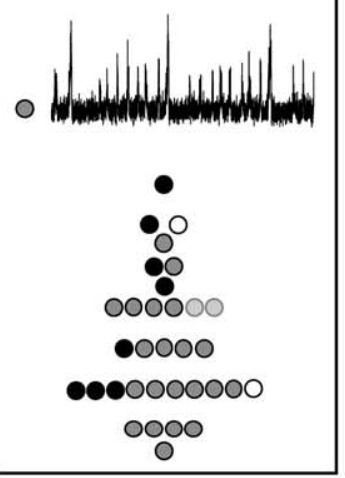

caudal boundary
$\mathrm{C}$

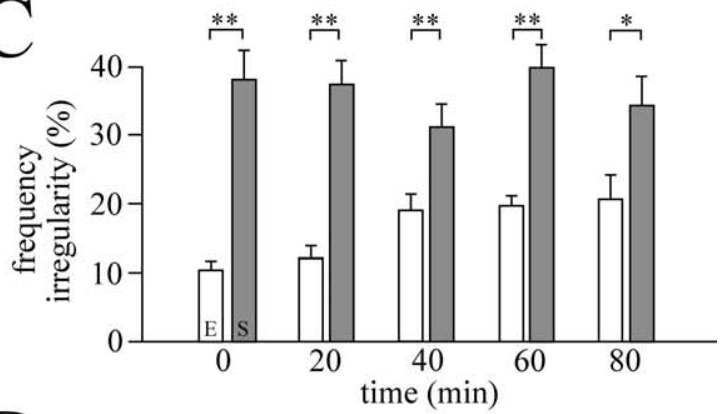

$\mathrm{D}$

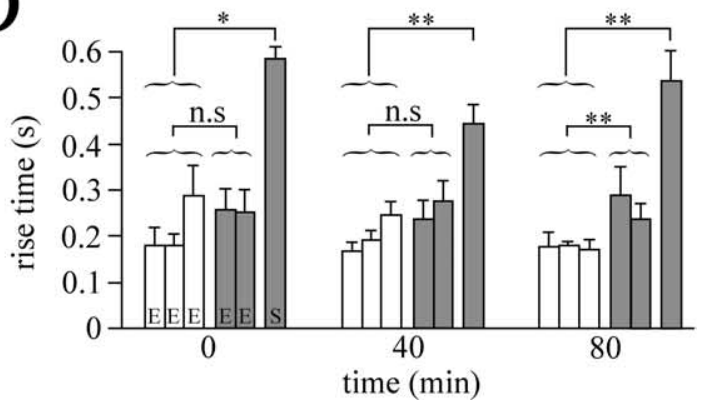

Figure 5. Rostrocaudal distribution and properties of inspiratory-related burst patterns in $3 \mathrm{~mm} \mathrm{~K}^{+}$of $700 \mu \mathrm{m}$ slices with unilaterally exposed preBötC. $A$, Plot of inspiratory-related patterns, specifically eupnea, eupnea- biphasic, and eupnea-sigh versus the rostral boundaries of $20 \mathrm{c}+$ preBötC [700/-0.44]S-D/W-P1/4 (blue box) and the caudal boundaries of $33 \mathrm{r}+$ preBötC[700/ $-0.60] \mathrm{S}-\mathrm{D} / \mathrm{W}-\mathrm{PO} / 3$ slices (purple box), respectively. Note that eupnea bursts had a rather small amplitude in some slices with a eupnea-sigh burst pattern (see example in top panel). Note also that the time scale for the example of a eupnea- biphasic burst pattern is expanded approximately threefold compared with the examples of rhythms with a solely eupnea or a eupnea-sigh pattern. $\boldsymbol{B}$, Irregularity score plots show that the burst amplitude of eupneic bursts $(\mathrm{E})$ in $\mathrm{c}+$ preBötc[700] (blue panel; $n=8$ ) slices was more uniform than that of sighs plus eupneic bursts $(\mathrm{S})$ in the $r+\operatorname{preBöt} C[700]$ slices (purple panel; $n=8)$. $C$, Also the frequency of eupneic bursts $(E)$ in the $c+$ preBötC $[700]$ slices of $B$ was significantly more regular than that of the eupnea-sigh bursts $(S)$ in the $r+$ preBötC[700] slices. In $\boldsymbol{B}$ and $\boldsymbol{C}$, significances were determined between mean values for each group at a given time period between 0 and 80 min of recording. $\boldsymbol{D}$, Plots show that the rise times of means of three consecutive eupneic bursts (E) in the $c+$ preBötC[700] slices do not differ from the means of two consecutive E bursts in the $r+$ preBötC[700] slices (except at 80 min) but are significantly shorter than the mean rise times of sighs (S) in the latter slices. Significance values are as follows: ${ }^{*} p<0.05 ;{ }^{* *} p<0.01 ;$ n.s., nonsignificant difference.

sigh burst pattern in 33 of 44 preBötC neurons that showed a similar pattern of $\mathrm{Ca}^{2+}$ rises before in vitro apnea. The other 11 cells also did not respond subsequently to $6-9 \mathrm{mM} \mathrm{K}^{+}$, again suggesting photodamage. Importantly, after the washout of GR73632 and activation of eupnea with rolipram, $\mathrm{Ca}^{2+}$ oscillations of uniform amplitude and frequency were evoked in the same 33 neurons that were active during the eupnea-sigh activity induced by GR73632 (Fig. 7B).

\section{Discussion}

The rostrocaudal extents of neuronal networks sufficient and necessary for generation of inspiratory-related behaviors were identified in newborn rat brainstem slices in $3 \mathrm{~mm}\left[\mathrm{~K}^{+}\right]$. The findings suggest that a small kernel of inspiratory preBötC neurons reconfigures in favor of a eupnea-sigh pattern via regions rostral to the preBötC or transmitters acting like SP, whereas it reconfigures in favor of a eupnea pattern via caudal regions or transmitters acting like TRH.

\section{A closer look at the preBötC}

The original report indicated that the preBötC extends $\sim 225 \mu \mathrm{m}$ rostrocaudally in newborn rats (Smith et al., 1991). Most studies since then used slices $\geq 500 \mu \mathrm{m}$ thick in elevated $\mathrm{K}^{+}$to facilitate motor output via hypoglossal nerve roots (Richter and Spyer, 2001; Ramirez et al., 2002). Slice boundaries that are necessary or sufficient for generating inspiratory rhythm have not been defined precisely. The original report in rats (Smith et al., 1991) and subsequent work in mice (Tryba et al., 2003) demonstrated that slices $>500 \mu \mathrm{m}$ thick generate inspiratory-related bursts in $3 \mathrm{~mm}$ $\mathrm{K}^{+}$, but only recently has it been shown that this behavior is stable for several hours (Ruangkittisakul et al., 2006). Here, we show that $250 \mu \mathrm{m}$ and $50 \%$ of $200-\mu \mathrm{m}$-thin slices, containing a common $(\sim 170 \mu \mathrm{m})$ portion of the preBötC, also generate robust bilaterally synchronized, inspiratory-related rhythm for $>1.5 \mathrm{~h}$ in $3 \mathrm{mM} \mathrm{K}^{+}$. The other $50 \%$ of $200 \mu \mathrm{m}$ slices produced bilaterally asynchronous rhythm. Furthermore, two of seven slices with $175 \mu \mathrm{m}$ thickness slices generated either bilaterally asynchronous or unilateral activity. The lack of bilateral (synchronous) bursting in the thinnest slices could reflect uneven ipsilateral and contralateral sectioning or tissue impairment by the suction electrode, which both would weaken neuronal connectivity unilaterally and between the bilaterally distributed preBötC. This would reduce recurrent excitation within this net- 
A

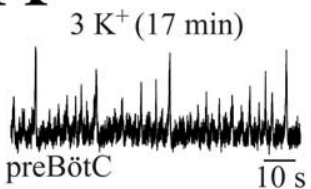

NK1 antagonist $(2.5 \mathrm{uM})$

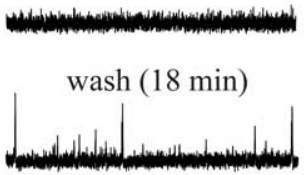

C $3 \mathrm{~K}^{+}(12 \mathrm{~min})$

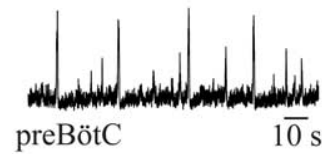

$\mathrm{E}$

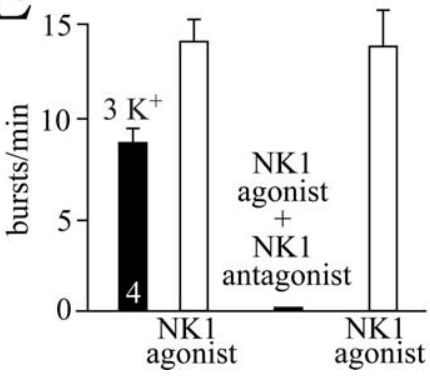

B

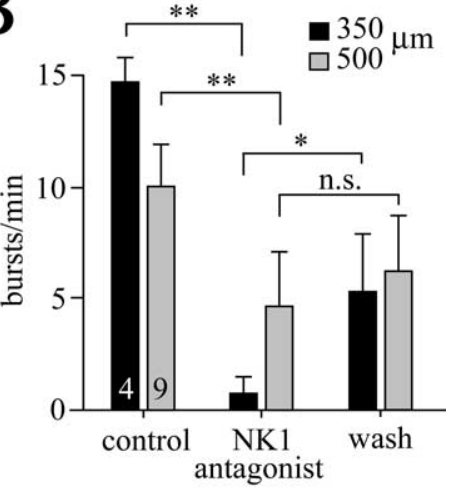

$\mathrm{D}$ $(5 \mathrm{nM})$

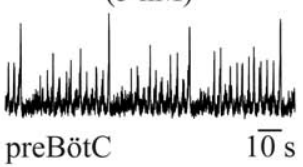

NK1 agonist + NK1 antagonist

$(2.5 \mu \mathrm{M})$
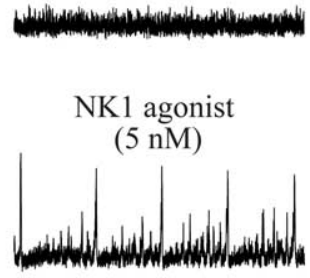

Figure 6. Depression of preBötC rhythms by block of NK1 receptors. $A$, Rhythm in $3 \mathrm{~mm} \mathrm{~K}^{+}$ with a eupnea-sigh burst pattern in a m-preBötC[350/-0.69]W-P1 slice was abolished 12 min after the start of bath application of the NK1 receptor antagonist GR82334. Recovery of the rhythm was incomplete within 18 min after the start of the washout of GR82334. B, Statistical analysis of the effects of the NK1 antagonist $(2.5 \mu \mathrm{m})$ on nine m-preBötC[500/- 0.75$] \mathrm{W}-\mathrm{P} 1 / 4$ slices (gray bars) and four m-preBötC[350/-0.70]W-P1/2 slices (black bars). Note that the block was more pronounced in the thinner slices. $C, \ln 3 \mathrm{~mm} \mathrm{~K}^{+}$, a m-preBötC[500/-0.68]W$\mathrm{PO}$ slice generated rhythm with a eupnea-sigh burst pattern. $\boldsymbol{D}$, After onset of in vitro apnea in the slice of $C$, bath application of the NK1 receptor agonist GR73632 restored a very similar eupnea-sigh burst pattern. GR82334 abolished the agonist-evoked rhythm within 4 min, whereas the rhythm was fully restored $10 \mathrm{~min}$ after washout of the NK1 antagonist. $\boldsymbol{E}$, Statistical analysis of the depressing effects of GR82334 (2.5 $\mu \mathrm{M})$ on the rhythms evoked by GR73632 (5 nM) in a group consisting of two m-preBötC[350/-0.70]W-P0 and two m-preBötC[500/ $-0.74]$ W-P2 slices. $\operatorname{In} \boldsymbol{B}$ and $\boldsymbol{E}$, digits in bars indicate the number of slices.

work below the threshold for synchronized burst generation (Del Negro et al., 2002a,b). As slice thickness is reduced, rhythmic burst amplitude decreases, presumably reflecting that fewer neurons are discharging in synchrony. Therefore, unilateral or no activity in slices $<200 \mu \mathrm{m}$ may indicate a limited sensitivity of suction electrode recording. Nevertheless, we can conclude that the preBötC core sufficient for the generation of inspiratory rhythm is, at most, $175 \mu \mathrm{m}$ in neonatal rats (Fig. 8). The preBötC kernel necessary for rhythm generation is notably smaller $(<100$ $\mu \mathrm{m}$, centered at $0.5 \mathrm{~mm}$ caudal to $\mathrm{VII}_{\mathrm{c}}$ ) when included with neighboring tissue (Fig. 8). A more exact determination of this region is limited by the resolution of on-line histology, possibly in conjunction with variation between rats in the extension of respiratory marker nuclei. Important to note, drive from neighboring tissue in $700-\mu \mathrm{m}$-thick slices results in a robust burst amplitude, even when one of their boundaries is $<100 \mu \mathrm{m}$ distant to the

center of the preBötC. That these rhythms in $3 \mathrm{mM} \mathrm{K}^{+}$are inspiratory related, whether recorded unilaterally or bilaterally, is based on the observation that they disappear if the recording suction electrode is moved by a few hundred micrometers from the hot spot that is colocated with the preBötC in the ventrolateral aspect of the slice surface (supplemental Fig. S2, available at www.jneurosci.org as supplemental material).

\section{A reconfiguring preBötC kernel}

All (except photodamaged) inspiratory preBötC neurons were active during $\mathrm{Ca}^{2+}$ imaging of both eupnea and eupnea-sigh burst patterns, supporting the hypothesis of Lieske et al. (2000) that multiple inspiratory behaviors derive from one reconfiguring network. In extension of their findings in $>500 \mu \mathrm{m}$ mouse slices, we show that inspiratory networks as thin as $250 \mu \mathrm{m}$ reconfigure under influence of specific neuromodulators (see below), indicating that this may be an intrinsic capacity of the preBötC kernel.

\section{Origin of sighs versus biphasic bursts}

The analysis of m-preBötC[500] slices showed that sighs and biphasic bursts can be distinguished by criteria such as amplitude, rise time, and the presence or absence of a second peak (supplemental material, available at www.jneurosci.org). However, they also share several features because their durations and rise times are both longer compared with eupneic bursts. A discrimination between these events is even more difficult in thin $\mathrm{m}$-preBötC slices. On the one hand, the fact that the primary burst type in both m-preBötC[250] and m-preBötC[200] slices is substantially larger and longer than intermittent eupnea-like bursts suggests that it is a sigh-like event. On the other hand, the shape of the larger-amplitude burst in the 250- $\mu \mathrm{m}$-thin slices resembles that of a sigh, whereas in the $200 \mu \mathrm{m}$ slices the shape is more consistent with a biphasic burst. It is possible that sighs and biphasic bursts constitute variations of one type of "augmented in vitro breath." Some yet unknown factors may determine whether the onset of the secondary event is earlier and its amplitude more pronounced (in the case of sighs), or whether its onset is delayed and of smaller amplitude (in the case of biphasic bursts). In line with this view, an initial eupnea-biphasic burst pattern could spontaneously transform into a eupnea-sigh pattern (supplemental Fig. S3B, available at www.jneurosci.org as supplemental material). It remains to be determined whether the occurrence of sighs versus biphasic bursts depends, for example, on (recurrent) mechanisms intrinsic to the emerging network of the preBötC kernel (Del Negro et al., 2002a,b).

\section{Spatiochemical organization of inspiratory networks}

A eupnea or a eupnea-sigh burst pattern was typical in $3 \mathrm{mM} \mathrm{K}^{+}$ for $700 \mu \mathrm{m}$ slices lacking either tissue rostral or caudal to the preBötC, respectively. We hypothesize that these distinct inspiratory-related patterns reflect a spatial organization of the medullary inspiratory network. Structures caudal to the preBötC kernel appear to promote eupnea, whereas areas rostral to the kernel appear to promote sighs (Fig. 8). These influences could derive from particular patterns of synaptic input including tonic drive, or specific neuromodulatory inputs as suggested by the differing effects of SP and TRH. Several brainstem structures neighboring the preBötC could account for this slice-typespecific behavior. For example, c+preBötC[700] and $\mathrm{r}+$ preBötC[700] slices may differ in the content of raphe obscurus versus raphe pallidus (Jacobs and Azmitia, 1992) that provide inputs to the ventral respiratory column (Holtman and Speck, 
A
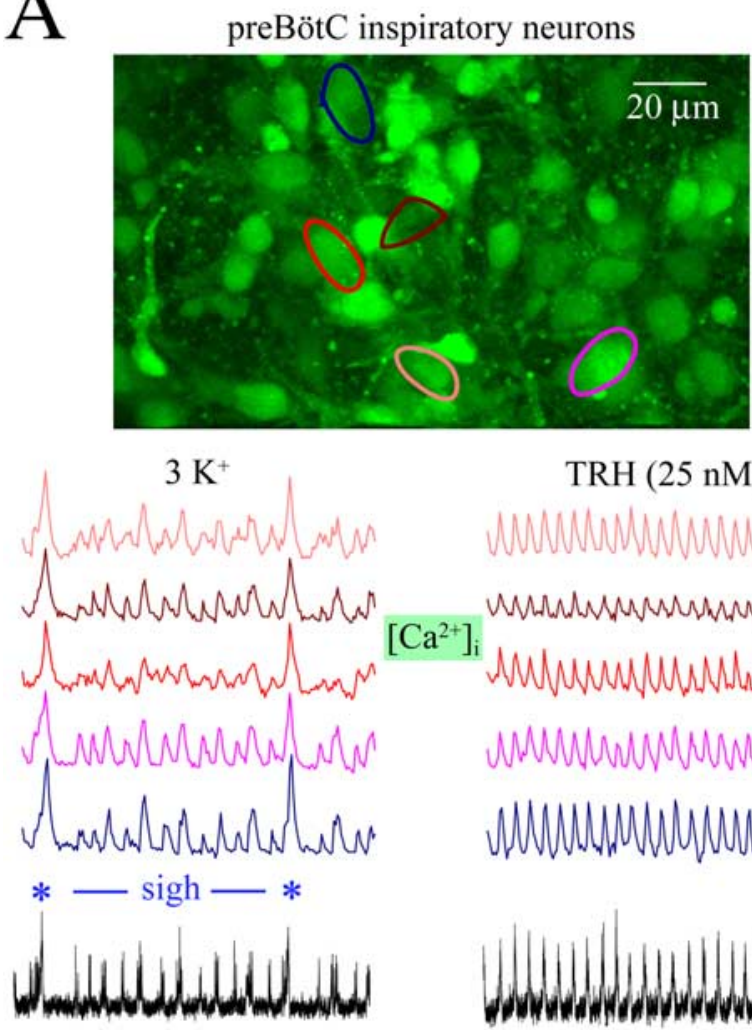

preBötC
B

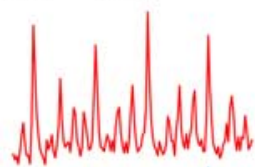

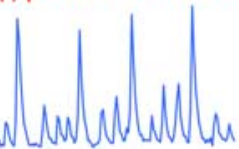

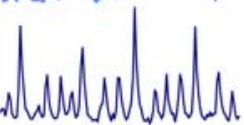

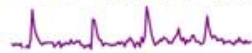

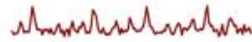

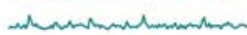

* * * * $*$

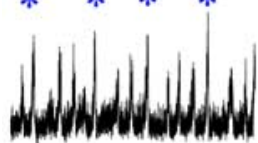

NK1 agonist

$(5 \mathrm{nM})$

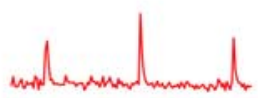

$\left[\mathrm{Ca}^{2+}\right]_{\mathrm{i}}$
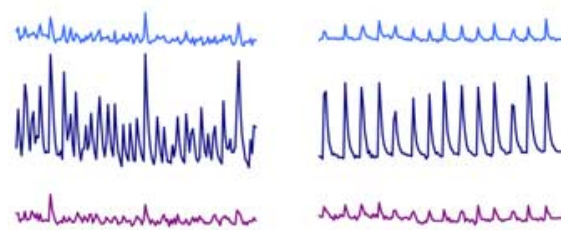

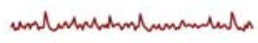

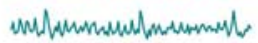
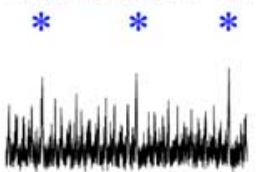

preBötC

(1 $\mu \mathrm{M})$
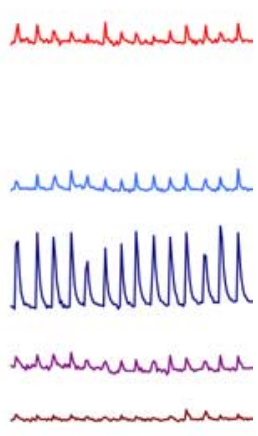

MAMUNGMUNA

$\overline{20 \mathrm{~s}}$

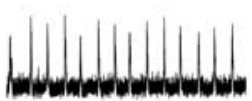

TRH (25 nM)

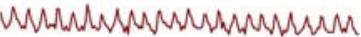

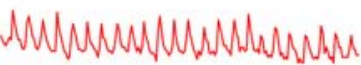

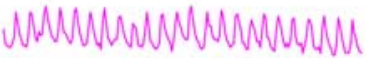

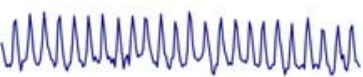
$\overline{10 \mathrm{~s}}$

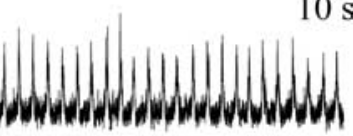

Rolipram

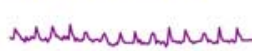

Figure 7. Reconfiguring burst patterns of inspiratory preBötC neurons visualized with multiphoton imaging of cytosolic $\left[\mathrm{Ca}^{2+}\right]$ rises. Cells were loaded with the membrane-permeant, $\mathrm{Ca}^{2+}$-sensitive dye fluo-4-AM by unilateral pressure injection into the preBötC (Ruangkittisakul et al., 2006). $\boldsymbol{A}_{1}\left[\mathrm{Ca}^{2+}\right]$ rises in preBötC neurons located $25 \mu \mathrm{m}$ caudal to the preBötC center occurred simultaneously with contralateral preBötC neuronal population activity recorded electrophysiologically from a $\mathrm{r}+$ preBötC[700/-0.58]W-P1 slice. Bath-applied TRH transformed both the inspiratory optical and electrophysiological pattern with two major sighs (asterisks) and various eupneic bursts into a more regular eupnea burst pattern without sighs. (See supplemental material, available at www.jneurosci.org, for movie files of dynamic $\mathrm{Ca}^{2+}$ changes and three-dimensional animation of neuronal morphology.) $\boldsymbol{B}$, The size of larger-

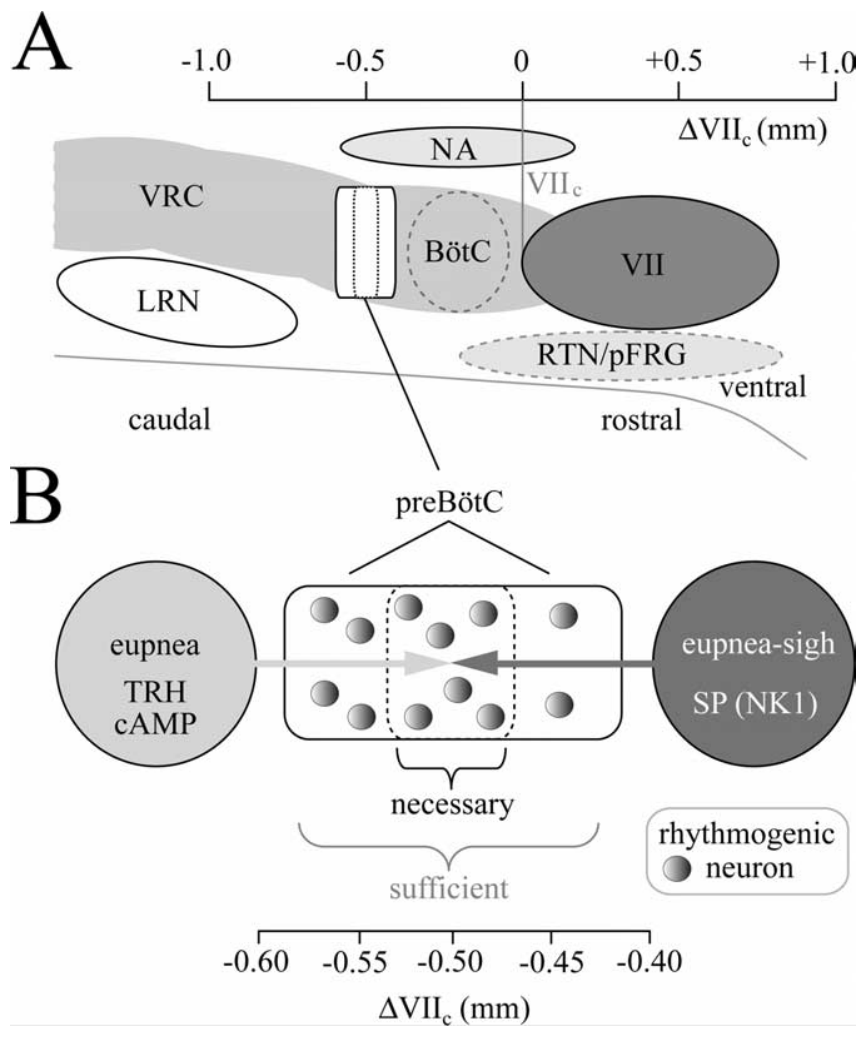

Figure 8. Organization of the rhythmogenic preBötC inspiratory network isolated in $3 \mathrm{~mm}$ $\mathrm{K}^{+} . \boldsymbol{A}$, Respiratory (marker) regions in a schematic sagittal section of a newborn rat brainstem. The scale is referred to $\mathrm{VII}_{c}$ [modified after Ruangkittisakul et al. (2006)]. For abbreviations, see Figure $1 B$. A summary of the main findings is as follows (see $\boldsymbol{B}$ ). The rostrocaudal extension of the rhythmogenic preBötC kernel sufficient for generation of inspiratory rhythm in thin brainstem slices is, at most, $175 \mu \mathrm{m}$, corresponding to the minimal thickness of slices with rhythmic activity in physiological $(3 \mathrm{~mm}) \mathrm{K}^{+}$solution. In contrast, the extension that is necessary for rhythm in $3 \mathrm{~mm} \mathrm{~K}^{+}$is possibly $<100 \mu \mathrm{m}$ in $700-\mu \mathrm{m}$-thick slices with the preBötC exposed at one boundary. The latter type of slices with the preBötC exposed to the rostral boundary generate rhythm resembling eupnea in vivo when receiving modulatory drive from caudal structures via a neurotransmitter(s) that acts like TRH and/or acts via increasing cAMP. Conversely, $700 \mu \mathrm{m}$ slices with the preBötC at the caudal surface generate an inspiratory pattern with a eupnea-sigh burst pattern provided by drive from structures rostral to the preBötC via a neurotransmitter(s) acting like SP on NK1 receptors. The rhythmogenic neurons of the preBötC kernel reconfigure their activity pattern after changes in the dominance of either drive.

1994). In raphe neurons, SP and TRH colocate with other modulators, but not with each other (Kachidian et al., 1991). Thus, their inputs to the preBötC may be organized in a rostrocaudal $\mathrm{SP}-\mathrm{TRH}$ gradient. The ventral respiratory column is also organized asymmetrically with regard to the preBötC, with the Bötzinger complex and the parafacial respiratory group/retrotrapezoid nucleus complex located rostrally and the rostral ventral respiratory group located caudally (Alheid et al., 2004). These or others structures, or neuromodulators, may reconfigure the preBötC kernel to favor one type of burst pattern (Richerson, 2004; Feldman and Del Negro, 2006; Pace et al., 2007). Although the mechanisms remain unknown, the hypothesized input from ros-

amplitude sigh (asterisks) and smaller-amplitude eupneic bursts recorded electrophysiologically from a $\mathrm{r}+$ preBötC[700/-0.60]W-P1 slice was reflected in the amplitude of $\mathrm{Ca}^{2+}$ oscillations in preBötC neurons located $60 \mu \mathrm{m}$ caudal to the preBötC center. After in vitro apnea, more irregular activities associated with a eupnea-sigh burst pattern were reactivated by the NK1 receptor agonist GR73632, whereas the CAMP-elevating blocker of phosphodiesterase-4 rolipram activated transients associated with eupneic bursts after washout of NK1 agonist. 
trally neighboring structures increases the probability at which the preBötC kernel produces sighs, which are typically followed by an inhibitory period as in mouse slices (Lieske et al., 2000; Telgkamp et al., 2002).

The importance of endogenous neuromodulatory drive in generating the typical pattern of sigh-like bursts and smaller eupnea bursts in the $200-250 \mu \mathrm{m}$ slices is not known. Possible sources are axotomized synaptic terminals or raphe cells. That a modulator can specifically influence slice activity is clear because both SP and NK1 agonist induced, after in vitro apnea, the eupnea-sigh burst pattern in all slices $\geq 250 \mu \mathrm{m}$ thick. More significant is that NK1 receptor antagonist depressed endogenous activity in $3 \mathrm{mM} \mathrm{K}^{+}$. We therefore hypothesize that SP is the transmitter of the rostral structures that promote a eupnea-sigh burst pattern. This view is consistent with previous findings in mouse slices in $8 \mathrm{~mm} \mathrm{~K}^{+}$that a NK1 receptor antagonist depressed inspiratory rhythm (Telgkamp et al. 2002), whereas SP increased the probability of sighs more than that of eupneic bursts (Lieske et al., 2000) and also restored rhythm during in vitro apnea (Pena and Ramirez, 2004). This supports the proposed importance of NK1 receptors for modulation of inspiratory rhythm (Murakoshi and Otsuka, 1985; Rampin et al., 1993; Gray et al., 1999, 2001; Morgado-Valle and Feldman, 2004).

In contrast to NK1 receptor activation, TRH (Hedner et al., 1983; Funk et al., 1994) reactivated a eupnea burst pattern in slices $\geq 250 \mu \mathrm{m}$ thick during in vitro apnea. A direct involvement of TRH in rhythm generation was not tested because of lack of antagonists. Eupnea was also induced by the clinical phosphodiesterase-4 blockers rolipram and theophylline (Fredholm et al., 1999; O’Donnell and Zhang, 2004; Ruangkittisakul and Ballanyi, 2006), supporting the idea that cAMP stimulates the preBötC (Ballanyi et al., 1997, 1999; Richter et al., 1997; Ballanyi, 2004). This also indicates that neurotransmitters that raise cellular cAMP such as serotonin via $5-\mathrm{HT}_{4(\mathrm{a})}$ receptors (Richter et al., 1997; Manzke et al., 2003), configure the network in favor of eupnea. However, the mechanisms may be more complex because a membrane-permeant cAMP analog stimulated both eupneic and sigh bursts in mouse slices (Lieske and Ramirez, 2006b). Pharmacological tools such as strychnine or modulators of metabotropic glutamate receptors that target either sighs or eupnea in mouse slices (Lieske et al., 2000; Lieske and Ramirez, 2006a,b) may be useful for dissecting the inspiratory patterns in the $3 \mathrm{mM} \mathrm{K}^{+}$newborn rat preBötC slices.

\section{References}

Alheid GF, Milsom WK, McCrimmon DR (2004) Pontine influences on breathing: an overview. Respir Physiol Neurobiol 143:105-114.

Ballanyi K (2004) Neuromodulation of the perinatal respiratory network. Curr Neuropharmacol 2:221-243.

Ballanyi K, Lalley PM, Hoch B, Richter DW (1997) cAMP-dependent reversal of opioid- and prostaglandin-mediated depression of the isolated respiratory network in newborn rats. J Physiol (Lond) 504:127-134.

Ballanyi K, Onimaru H, Homma I (1999) Respiratory network function in the isolated brainstem-spinal cord of newborn rats. Prog Neurobiol 59:583-634.

Bhatia J (2000) Current options in the management of apnea of prematurity. Clin Pediatr (Phila) 39:327-336.

Del Negro CA, Wilson CG, Butera RJ, Rigatto H, Smith JC (2002a) Periodicity, mixed-mode oscillations, and quasiperiodicity in a rhythmgenerating neural network. Biophys J 82:206-214.

Del Negro CA, Morgado-Valle C, Feldman JL (2002b) Respiratory rhythm: an emergent network property? Neuron 34:821-830.

Feldman JL, Del Negro CA (2006) Looking for inspiration: new perspectives on respiratory rhythm. Nat Rev Neurosci 7:232-242.

Fredholm BB, Battig K, Holmen J, Nehlig A, Zvartau EE (1999) Actions of caffeine in the brain with special reference to factors that contribute to its widespread use. Pharmacol Rev 51:83-133.

Funk GD, Smith JC, Feldman JL (1993) Generation and transmission of respiratory oscillations in medullary slices: role of excitatory amino acids. J Neurophysiol 70:1497-1515.

Funk GD, Smith JC, Feldman JL (1994) Development of thyrotropinreleasing hormone and norepinephrine potentiation of inspiratoryrelated hypoglossal motoneuron discharge in neonatal and juvenile mice in vitro. J Neurophysiol 72:2538-2541.

Gray PA, Rekling JC, Bocchiaro CM, Feldman JL (1999) Modulation of respiratory frequency by peptidergic input to rhythmogenic neurons in the preBötzinger complex. Science 286:1566-1568.

Gray PA, Janczewski WA, Mellen N, McCrimmon DR, Feldman JL (2001) Normal breathing requires preBötzinger complex neurokinin-1 receptorexpressing neurons. Nat Neurosci 4:927-930.

Hedner J, Hedner T, Wessberg P, Lundberg D, Jonason J (1983) Effects of TRH and TRH analogues on the central regulation of breathing in the rat. Acta Physiol Scand 117:427-437.

Holtman Jr JR, Speck DF (1994) Substance P immunoreactive projections to the ventral respiratory group in the rat. Peptides 15:803-808.

Jacobs BL, Azmitia EC (1992) Structure and function of the brain serotonin system. Physiol Rev 72:165-229.

Kachidian P, Poulat P, Marlier L, Privat A (1991) Immunohistochemical evidence for the coexistence of substance $\mathrm{P}$, thyrotropin-releasing hormone, GABA, methionine-enkephalin, and leucin-enkephalin in the serotonergic neurons of the caudal raphe nuclei: a dual labeling in the rat. J Neurosci Res 30:521-530.

Lieske SP, Ramirez JM (2006a) Pattern-specific synaptic mechanisms in a multifunctional network. I. Effects of alterations in synapse strength. J Neurophysiol 95:1323-1333.

Lieske SP, Ramirez JM (2006b) Pattern-specific synaptic mechanisms in a multifunctional network. II. Intrinsic modulation by metabotropic glutamate receptors. J Neurophysiol 95:1334-1344.

Lieske SP, Thoby-Brisson M, Telgkamp P, Ramirez JM (2000) Reconfiguration of the neural network controlling multiple breathing patterns: eupnea, sighs and gasps. Nat Neurosci 3:600-607.

Manzke T, Guenther U, Ponimaskin EG, Haller M, Dutschmann M, Schwarzacher S, Richter DW (2003) 5-HT4(a) receptors avert opioid-induced breathing depression without loss of analgesia. Science 301:226-229.

Morgado-Valle C, Feldman JL (2004) Depletion of substance P and glutamate by capsaicin blocks respiratory rhythm in neonatal rat in vitro. J Physiol (Lond) 555:783-792.

Mulkey DK, Stornetta RL, Weston MC, Simmons JR, Parker A, Bayliss DA, Guyenet PG (2004) Respiratory control by ventral surface chemoreceptor neurons in rats. Nat Neurosci 7:1360-1369.

Murakoshi T, Otsuka M (1985) Respiratory reflexes in an isolated brainstem-lung preparation of the newborn rat: possible involvement of $\gamma$-aminobutyric acid and glycine. Neurosci Lett 62:63-68.

O'Donnell JM, Zhang HT (2004) Antidepressant effects of inhibitors of cAMP phosphodiesterase (PDE4). Trends Pharmacol Sci 25:158-163.

Onimaru H, Homma I (2003) A novel functional neuron group for respiratory rhythm generation in the ventral medulla. J Neurosci 23:1478-1486.

Pace RW, Mackay DD, Feldman JL, Del Negro CA (2007) Inspiratory bursts in the preBötzinger complex depend on a calcium-activated nonspecific cationic current linked to glutamate receptors. J Physiol (Lond) 582:113-125.

Pena F, Ramirez JM (2004) Substance P-mediated modulation of pacemaker properties in the mammalian respiratory network. J Neurosci 24:7549-7556.

Ramirez JM, Zuperku EJ, Alheid GF, Lieske SP, Ptak K, McCrimmon DR (2002) Respiratory rhythm generation: converging concepts from in vitro and in vivo approaches? Respir Physiol Neurobiol 131:43-56.

Rampin O, Pierrefiche O, Denavit-Saubie M (1993) Effects of serotonin and substance $\mathrm{P}$ on bulbar respiratory neurons in vivo. Brain Res 622:185-193.

Rekling JC, Feldman JL (1998) PreBötzinger complex and pacemaker neurons: hypothesized site and kernel for respiratory rhythm generation. Annu Rev Physiol 60:385-405.

Richerson GB (2004) Serotonergic neurons as carbon dioxide sensors that maintain pH homeostasis. Nat Rev Neurosci 5:449-461. 
Richter DW, Spyer KM (2001) Studying rhythmogenesis of breathing: comparison of in vivo and in vitro models. Trends Neurosci 24:464-472.

Richter DW, Lalley PM, Pierrefiche O, Haji A, Bischoff AM, Wilken B, Hanefeld V, (1997) Intracellular signal pathways controlling respiratory neurons. Respir Physiol Neurobiol 110:113-123.

Ruangkittisakul A, Ballanyi K (2006) Reversal by phosphodiesterase-4 blockers of in vitro apnea in the isolated brainstem-spinal cord preparation from newborn rats. Neurosci Lett 401:194-198.

Ruangkittisakul A, Schwarzacher SW, Secchia L, Poon BY, Ma Y, Funk GD, Ballanyi K (2006) High sensitivity to neuromodulator-activated signaling pathways at physiological $\left[\mathrm{K}^{+}\right]$of confocally imaged respiratory center neurons in on-line-calibrated newborn rat brainstem slices. J Neurosci 26:11870-11880.

Shvarev YN, Lagercrantz H, Yamamoto Y (2003) Two types of rhythm in the respiratory network output in the isolated ventrolateral medulla in the neonatal rats. Neurosci Lett 347:53-56.

Smith JC, Ellenberger HH, Ballanyi K, Richter DW, Feldman JL (1991) PreBötzinger complex: a brainstem region that may generate respiratory rhythm in mammals. Science 254:726-729.

Telgkamp P, Cao YQ, Basbaum AI, Ramirez JM (2002) Long-term deprivation of substance P in PPT-A mutant mice alters the anoxic response of the isolated respiratory network. J Neurophysiol 88:206-213.

Tryba AK, Pena F, Ramirez JM (2003) Stabilization of bursting in respiratory pacemaker neurons. J Neurosci 23:3538-3546.

Tryba AK, Pena F, Ramirez JM (2006) Gasping activity in vitro: a rhythm dependent on 5-HT2A receptors. J Neurosci 26:2623-2634.

Yuste R, Konnerth A, Masters BR (2006) Imaging in neuroscience and development, a laboratory manual. J Biomed Opt 11:19902. 